\title{
Monday Poster Session
}

\section{The Wilson Suite}

\section{$4 \mathrm{pm}$ to $6 \mathrm{pm}$}

Abstracts in board number order

Please ensure you display your poster from $3.30 \mathrm{pm}$ to $6 \mathrm{pm}$.

Moderation will be ongoing during the poster session. Please ensure you stay with your poster during this time.

Any posters left at the end of the day will be removed. 
Board: 1

Title: Distinct changes in cerebral artery structure distal and proximal to the lesion site in ischemic stroke

Authors: Shaun L Sandow, ${ }^{1,2}$ Nicole M Jones, ${ }^{3}$ Nathan Luque, ${ }^{1}$ T Hilton Grayson, ${ }^{1}$ Hong Nguyen, ${ }^{3}$ Timothy V Murphy ${ }^{2}$

Address: $\quad{ }^{1}$ Inflammation and Healing Cluster, Faculty of Science, Health, Education and Engineering, University of the Sunshine Coast, Sippy Downs, Qld, Australia; ${ }^{2}$ Physiology, ${ }^{3}$ Pharmacology, School of Medical Sciences, University of NSW, Sydney, NSW Australia

Abstract: Occlusion of the middle-cerebral artery (MCA) underlies ischemic stroke (IS). Understanding fundamental mechanisms of cerebral blood flow, and identifying and developing strategies to target and control these is critical for the development of IS therapy. Caveolae are sites of integrated microdomain signalling, as key organelles for the modulation of blood flow in health and disease. This study determines vessel wall morphology and endothelial caveolae density distal and proximal to the lesion site in IS. Vessel wall morphology and caveolae density are hypothesized to be altered in IS.

Ischemic stroke was induced in 8-10 week male Sprague Dawley rats via bolus endothelin-1 (120 pM) injection to the central MCA. After behavioural grading and 5 days recovery, animals were perfusion fixed for TEM and vessel segments processed and imaged at $\times 0.8-30 k$. Data were analyzed per previous studies.

\begin{tabular}{lllll}
\hline \multicolumn{3}{l}{ dMCA } & \multicolumn{1}{l}{ pMCA } \\
\hline cohort and ' $n$ ' & control (3) & stroke (4) & control (3) & stroke (3) \\
\hline diameter $(\mu \mathrm{m})$ & $139 \pm 6$ & $146 \pm 9$ & $259 \pm 9$ & $255 \pm 11$ \\
number SMC layers & $2.9 \pm 0.2$ & $2.0 \pm 0.2 *(\downarrow \sim 30 \%)$ & $2.9 \pm 0.2$ & $3.0 \pm 0.3$ \\
wall thickness $(\mu \mathrm{m})$ & $13.6 \pm 0.1$ & $7 \pm 1.2 *(\downarrow \sim 50 \%)$ & $9.7 \pm 0.7$ & $10.2 \pm 0.6$ \\
lumenal caveolae density & $1.6 \pm 0.1$ & $2.9 \pm 0.2 *(\uparrow \sim 80 \%)$ & $5.7 \pm 0.1$ & $4.8 \pm 0.2 *(\downarrow \sim 16 \%)$ \\
$(/ \mu \mathrm{m})$ & & & \\
$\begin{array}{l}\text { ablumenal caveolae } \\
\text { density }(/ \mu \mathrm{m})\end{array}$ & $1.1 \pm 0.1$ & $1.4 \pm 0.1$ & $2.2 \pm 0.1$ & $1.7 \pm 0.2$ \\
\hline Mean $\pm \mathrm{SEM} ; * P<0.05$, as significant $c f /$. control. ' $n$ ', each from a different animal.
\end{tabular}

Remodelling of MCA differs in vessel segments distal and proximal to the IS lesion site. Altered caveolae density may reflect changes in specific microdomain function.

Determining the functional consequences and the nature of the stimulus for remodelling in specific vessel segments (shear stress/flow/pressure) is the subject of ongoing work. 
Board: 2

Title: $\quad$ SKA-31, an activator of endothelial KCa channels, is an effective vasodilator in isolated rat mesenteric arteries

Authors: Rayan Khaddaj Mallat, Andrew P Braun

Address: Dept. of Physiology and Pharmacology, Cumming School of Medicine, University of Calgary

Abstract: The vascular endothelium critically determines systemic blood pressure and tissue blood flow by regulating the intraluminal diameter of resistance arteries. Previous work from our group has demonstrated that endothelial Ca2+-activated $\mathrm{K}+(\mathrm{KCa})$ channels are critical "drivers" of agonist-evoked endothelial hyperpolarization, NO production, vasodilation and augmented coronary flow.

The goal of the present study is to investigate the actions of SKA-31, a small molecule activator of KCa 2.x and KCa 3.1 channels, in the mesenteric vascular bed, which receives up to a third of cardiac output and contributes to total peripheral resistance. Specifically, we have examined the mode of action of SKA-31 to evoke and/or improve vasodilation of mesenteric arteries, isolated from male Sprague Dawley (SD) rats.

Animals (225-250 g body weight) were euthanized by intraperitoneal administration of sodium pentobarbital $(50 \mathrm{mg} / \mathrm{kg}$ ) and arteries (e.g. 200-400 $\mu \mathrm{m}$ intraluminal diameter) were isolated and cannulated in a pressure myography chamber. Arteries were pressurized to $70 \mathrm{mmHg}$, pre-constricted with $1 \mu \mathrm{M} \mathrm{PE}$ (phenylephrine) and superfused at a constant flow of $6-7 \mathrm{ml} / \mathrm{min}$ with Krebs' solution at $\sim 36^{\circ} \mathrm{C}$. Vasoactive agents were added directly to the superfusate.

SKA-31 and ACh produced robust vasodilation in arteries with intact endothelium that was abolished following endothelial denudation. These vasodilatory effects were largely prevented by the KCa2.3 blocker UCL1684 and the KCa3.1 blocker TRAM-34. In contrast, treatment with Penitrem-A, a blocker of smooth muscle BKCa channels, did not alter SKA-31 evoked vasodilation. Furthermore, the eNOS inhibitor L-NAME did not affect the inhibitory response to SKA-31, but the combined treatment of L-NAME + indomethacin (cyclooxygenase blocker) impaired vasodilation evoked by either ACh or SKA-31.

Collectively, these data demonstrate that SKA-31 acts in an endothelium-dependent manner to produce robust inhibition of PE pre-constricted mesenteric arteries exhibiting modest myogenic tone.

This work was supported by a research grant to APB from the Canadian Institutes of Health Research. 


\section{Board: 3}

Title: $\quad \mathrm{K}_{\mathrm{Ca}}$ channels modulate vascular superoxide anion production

Authors: S.E. Lunn ${ }^{1}$, P. M. Kerr² , F. Plane ${ }^{1}$.

Address: ${ }^{1}$ Department of Pharmacology, University of Alberta, Edmonton, AB., Canada

${ }^{2}$ Department of Nursing Science, MacEwan University, Edmonton, AB., Canada

Abstract: Background: The endothelium limits vasoconstriction through release of diffusible nitric oxide (NO), and spread of membrane potential hyperpolarization through gap junctions. Both pathways are activated by agonist-evoked smooth muscle contraction via InsP3-mediated myoendothelial feedback. NO levels are regulated by both its production by $\mathrm{NO}$ synthase (NOS), and scavenging by superoxide anions $\left(\mathrm{O}_{2}{ }^{-}\right)$. In macrophages, the activity of NADPH oxidase, the main source of endothelium-derived $\mathrm{O}_{2}{ }^{-}$, can be regulated by changes in membrane potential. We have investigated if selective pharmacological activators of endothelial small (SKCa) and intermediate (IK $\mathrm{K}_{\mathrm{Ca}}$ ) conductance $\mathrm{Ca}^{2+}$-activated $\mathrm{K}^{+}$channels can reduce $\mathrm{O}_{2}{ }^{-}$and thus, enhance the ability of endothelium-derived NO to limit vasoconstriction.

Methods: Rat mesenteric arteries were mounted on a pressure myograph attached to an lonOptix system for simultaneous recording of changes in diameter and $\mathrm{O}_{2}{ }_{2}^{-}$. Vessels were maintained at $60 \mathrm{mmHg}$ and concentration-response curves to phenylephrine (PE; 0.01-10 $\mu \mathrm{M}$ ) were constructed in the presence and absence of selective pharmacological tools.

Results: Apocynin, a NADPH oxidase inhibitor and/or scavenger of $\mathrm{O}_{2}{ }^{-}$, significantly reduced both $\mathrm{PE}$ induced $\mathrm{O}_{2}$ production and constriction. The NOS inhibitor, L-NAME, had no effect on $\mathrm{PE}$-induced $\mathrm{O}_{2}$ production but significantly enhanced constriction. CyPPA, a positive modulator of $\mathrm{SK}_{\mathrm{Ca}}$ channels, significantly reduced $\mathrm{PE}$-induced $\mathrm{O}_{2}{ }^{-}$ production while SKA-31, a selective $\mathrm{IK}_{\mathrm{Ca}}$ channel activator, significantly limited both $P E$-induced $\mathrm{O}_{2}$ production and constriction. LNAME blocked the effects of both SKA-31 and CyPPA.

Conclusions: We show that positive modulators of endothelial $\mathrm{K}_{\mathrm{Ca}}$ channels reduce $\mathrm{O}_{2}$ levels and so may provide a novel approach to enhance NO availability. 


\section{Board: 4}

Title: Vascular Conduction Coordinates Cerebral Arterial Tone and Brain Blood Flow Control

Authors: Anil Zechariah ${ }^{1}$, Cam Ha T. Tran ${ }^{2}$, Bjorn O. Hald ${ }^{3}$, Ursula I. Tuor ${ }^{2}$, Tim Watson ${ }^{2}$, Grant R.J. Gordon ${ }^{2}$, Donald G. Welsh ${ }^{1,2}$

Address: $\quad{ }^{1}$ Robarts Research Institute and the Department of Physiology and Pharmacology University of Western Ontario, London, Ontario, Canada

${ }^{2}$ Hotchkiss Brain Institute, Libin Cardiovascular Institute and the Department of Physiology and Pharmacology, University of Calgary, Alberta, Canada

${ }^{3}$ Department of Biomedical Sciences, University of Copenhagen, Denmark

Abstract: Cerebral arterial networks are tasked with the responsibility of matching neural activity with blood flow delivery. At the foundational level, neurovascular coupling is in essence a two step process, with a stimulus generating a focal response which secondarily spreads to initiate the broader changes in vascular resistance needed to facilitate perfusion. Spreading responses are intrinsic to the arterial wall and require a common signal to be shared among vascular cells. This study demonstrated using a complement of approaches that charge is that common signal and its conduction along the endothelium and then to the smooth muscle layer, coordinates contractile activity in the cerebral arterial network. Conduction is dependent on gap junctional expression and as in silco work illustrates, the structural/vascular properties of vascular cells are optimized to enable electrical and the corresponding vasomotor response to spread from penetrating arterioles to the surface vessel. Ascending responses were functionally observed in during whisker stimulation and they were strikingly impaired in mice lacking Cx40, a key gap junctional subunit expressed in endothelial cells. Impairing the ability of response to ascend into cortical surface network compromised blood flow responses induced by stroke, facilitating brain injury. Conduction is an integral component of the neurovascular coupling and it is essential to optimize the blood flow delivery, albeit under actively dynamic or pathophysiological conditions. 


\section{Board: 5}

Title: $\quad$ Baroreflex sensitivity in rats with genetic and salt hypertension

Authors: Behuliak Michal, Zicha Josef

Address: Institute of Physiology, The Czech Academy of Sciences, Prague, Czech Republic

Abstract: The main purpose of the baroreceptor-heart rate (HR) reflex is to maintain blood pressure (BP) close to a particular set point over a relatively short period of time. Attenuation of the baroreflex sensitivity has been reported not only in many forms of experimental hypertension but also in human essential hypertension. Our study was aimed to determine baroreflex sensitivity in rats with three different forms of experimental hypertension - spontaneously hypertensive rats (SHR), Dahl high-salt (HS) hypertensive (DS-HS) rats and Ren-2 transgenic rats (TGR), as well as in their normotensive controls.

$\mathrm{BP}$ and $\mathrm{HR}$ responses to intravenous administration of vasodilator nitroprusside (1-100 $\mu \mathrm{g} / \mathrm{kg}$ ) and vasopressor phenylephrine (1-100 $\mu \mathrm{g} / \mathrm{kg}$ ) were measured in conscious rats. BP-responses were plotted against HR-responses and analyzed by a sigmoidal logistic function curve. Baroreflex sensitivity was calculated from the slopes of regression lines separately for sympathetic component (nitroprusside-induced MAP reduction and reflex tachycardia) and for vagal component (phenylephrine-induced MAP elevation and reflex bradycardia).

Conscious hypertensive rats (SHR, TGR and DS-HS rats) have elevated blood pressure and diminished sympathetic component of baroreflex sensitivity compared to their respective normotensive controls (Wistar-Kyoto, Sprague-Dawley or Dahl salt-resistant rats). SHR has also diminished vagal component (mainly due to its reduced maximum capacity). Vagal component of baroreceptor-HR reflex were also diminished in Dahl salt-sensitive rats compared to Dahl salt-resistant rats, irrespective of salt intake and/or BP elevation.

Baroreflex sensitivity is attenuated in all three forms of experimental hypertension. Both sympathetic and vagal components of baroreceptor-HR reflex are usually attenuated in rats with genetic predisposition to hypertension.

(Supported by grant GACR 16-10349Y) 
Board: 6

Title: $\quad$ Collagen recruitment at low pressure limits distension of human resistance arteries

Authors: Maria Bloksgaard ${ }^{1}$, Kristoffer Rosenstand ${ }^{1}$, Jonathan Brewer ${ }^{2}$, Koen Reesink $^{3}$, Lars Melholt Rasmussen ${ }^{4}$, Akhmadjon Irmukhamedov ${ }^{5}$, Jo G.R De Mey ${ }^{1,5}$

Address: University of Southern Denmark; ${ }^{1}$ Dept. Cardiovascular and Renal Research, Institute of Molecular and 'MEMPHYS-Center for Biomembrane Physics, Odense, Denmark

Maastricht University; 'Dept. Biomedical Engineering, Cardiovascular Research Institute Maastricht, Maastricht, the Netherlands

Odense University Hospital; 'Dept. Clinical Biochemistry and Pharmacology, and ${ }^{5}$ Dept. Cardiac, Thoracic and Vascular Surgery, Odense, Denmark

Abstract: Disease related changes in the extracellular matrix (ECM) and the influence hereof on mechanical properties of human resistance arteries largely remain to be determined. Human pericardial resistance arteries (PRA) display a different ECM microarchitecture compared to frequently used rodent mesenteric arteries. We hypothesized that the biaxial mechanics of PRA mirror pressure induced changes in the ECM microarchitecture.

Pig PRA, with an ECM microarchitecture resembling human PRA, were used to validate an integrated approach using vital imaging, pressure myography and mathematical modeling. The incremental elastic moduli $\mathrm{E}_{\text {inc }}$ of the arterial wall linearly relate to the straightness of adventitial collagenfibers. In contrast, there is a nonlinear relationship of $\mathrm{E}_{\text {inc }}$ to the internal elastic lamina fiber branching angles. Mathematical modeling yielded a mean (SE) collagen recruitment strain of $1.1(0.2)$ circumferentially and 0.20 (0.01) longitudinally, corresponding to a pressure of $\sim 40$ $\mathrm{mmHg}$, as corroborated by the imaging. Human PRA show limited circumferential distensibility and elongation and a collagen recruitment strain of $0.8(0.1)$ circumferentially and $0.06(0.02)$ longitudinally, reached at a distending pressure below $20 \mathrm{mmHg}$. This finding is confirmed by vital imaging showing negligible microarchitectural changes of elastin and collagen upon pressurization.

In conclusion, we show for the first time in resistance arteries a quantitative relationship between arterial wall mechanics and pressure-induced changes in the microarchitecture of the extracellular matrix. Furthermore, we show that collagen limits the distension of human pericardial resistance arteries from patients with cardiovascular diseases already at at pressure of $20 \mathrm{mmHg}$. The strength of the integrated methods invites for future detailed studies of selected microvascular pathologies. 
Board: 7

Title: $\quad$ Enhanced endothelial function in arteries from human colon cancer

Authors: Ninna Schmidt Voss ${ }^{1,2}$, Henrik H. Kold-Petersen ${ }^{3}$, Ida Holm ${ }^{4}$, Ebbe Boedtkjer ${ }^{2}$

Address: $\quad{ }^{1}$ Research Unit, Regional Hospital Randers, ${ }^{2}$ Department of Biomedicine, Aarhus University, ${ }^{3}$ Surgical Department, Regional Hospital Randers, ${ }^{4}$ Department of Pathology, Regional Hospital Randers, Denmark

\section{Abstract: Background:}

Insufficient perfusion of cancer tissue results in low local nutrient and $\mathrm{O} 2$ levels and buildup of acidic waste products. Tumor vascularization has been studied in some detail but our understanding of functional differences between cancer and normal arteries is inadequate.

Methods: Biopsies of human primary colon carcinomas and matched normal colon tissue-at least $10 \mathrm{~cm}$ from macroscopically identifiable tumor tissue-were sampled from operations at Regional Hospital Randers, immediately transferred to ice-cold physiological saline solution, and brought to Department of Biomedicine, Aarhus University. Cancer feed arteries and matched normal arteries of similar diameter were dissected from the respective biopsies and mounted in wire myographs for studies of tunica media thickness, vasomotion, vasoconstriction, and vasorelaxation.

Results: Vasoconstriction in response to endothelin-1 and thromboxane analog U46619 was attenuated in colon cancer feed arteries compared to control arteries whereas responses to arginine vasopressin and elevated extracellular $[\mathrm{K}+]$ were unaffected. Endothelium-dependent vasorelaxation to acetylcholine was enhanced in colon cancer feed arteries. The differences in vasorelaxant and vasocontractile function between colon cancer feed arteries and arteries from normal colon tissue decreased after inhibition of endothelial NO synthesis with L-NAME. We found no difference in the vasorelaxant response to NO-donors $S$-nitroso- $N$-acetylpenicillamine, sodium nitroprusside, and spermine NONOate or in the thickness of the arterial media. Vasomotion in colon cancer feed arteries had similar frequency but lower amplitude than in normal arteries.

\section{Conclusion:}

We propose that increased endothelial NO synthesis amplifies vasorelaxation and attenuates vasoconstriction of colon cancer feed arteries, which will facilitate tumor perfusion.

Formateret: Dansk 


\section{Board: 8}

Title: $\quad$ G protein-mediated signaling in the myogenic response - role of Rho-kinase and aging

Authors: Karl Björling, ${ }^{1,2, *}$ Philomeena Daphne Joseph, ${ }^{1,{ }^{*}}$ Kristian Egebjerg, ${ }^{1, *}$ Jakob Lerche Hansen, ${ }^{3}$ Max Salomonsson, ${ }^{2}$ and Lars Jørn Jensen ${ }^{1}$

* These authors contributed equally to the work.

Address: ${ }^{1)}$ Departments of Veterinary and Animal Sciences and ${ }^{2)}$ Biomedical Sciences, Faculty of Health and Medical Sciences, University of Copenhagen, Denmark; ${ }^{3)}$ Diabetes \& Cardiovascular Pharmacology, Novo Nordisk A/S, Måløv, Denmark

Abstract: A myogenic response (MR) is a reflex vasoconstriction to an increase in intraluminal pressure, which is crucial for autoregulation of tissue blood flow and basal, myogenic tone (MT) in resistance vessels. Development of MT is suggested to involve mechanical activation of $\mathrm{G}$ protein-coupled receptors. Aim: 1 ) to investigate the signaling events from $G_{q / 11}$ and/or $G_{12}$ activation to MT development; 2 ) to elucidate the impact of aging. We used pressure myography, calcium imaging, Q-PCR and immunofluorescence to study small (lumen $D<200 \mu \mathrm{m}$ ) mesenteric arteries (SMA) from young (2-3 months), mature adult (6-7 months), and old (12-13 months) wildtype (C57BL6) mice.

Theoretical blood flow calculations in SMA indicated autoregulation of blood flow at pressures from 60 to $120 \mathrm{~mm} \mathrm{Hg}$. $\mathrm{G}_{\mathrm{q} / 11}$ and $\mathrm{G}_{12}$ were abundantly expressed at the mRNA and protein levels in SMA, and the G匹/q inhibitor YM-254890 $(0.1 \mu \mathrm{M})$ suppressed MT development. The Phosholipase $C$ inhibitors U73122 $(0.5 \mu \mathrm{M})$ and ET18-OCH3 $(10 \mu \mathrm{M})$ robustly inhibited MT, and the TRPC channel blocker SKF 96365 $(10 \mu \mathrm{M})$ slightly reduced MT. There was a significant effect of age $(P<0.0001)$ with MT being increased in mature adult mice and reduced in old mice compared to young mice. The ROCK2 inhibitor KD025 (5 $\mu \mathrm{M})$ robustly inhibited MT in all mice, but this effect was significantly larger in mature adult mice. Our data suggest that MT development in SMAs is initiated by phosphorylation of Myosin Light Chain through a

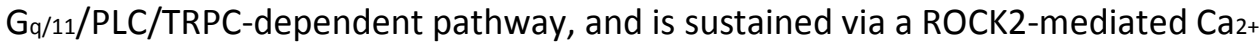
sensitization. Increased MT at mature adulthood may be explained by increased ROCK2 activity. 
Board: 9

Title: $\quad$ Consequences of $\mathrm{Na}+, \mathrm{HCO} 3-$-cotransporter $\mathrm{NBCn} 1$ for vascular smooth muscle cells and resistance artery function

Authors: Nicolai Jessen, Jakob Vaarst, Christian Aalkjaer and Ebbe Boedtkjer

Address: Department of Biomedicine, Aarhus University, Denmark

Abstract: Blood pressure regulation is disturbed in several animal models with disrupted expression of acid-base transporters; and reciprocally, disturbed acid-base transport function has been described in hypertensive individuals. $\mathrm{Na}+, \mathrm{HCO} 3-$-cotransporter NBCn1 represents the main mechanism of net acid extrusion from vascular smooth muscle and endothelial cells of resistance arteries. The overall vasocontractile response to noradrenaline is unaffected in resistance arteries from mice with global disruption of NBCn1; however, when the vasodilatory influence of the endothelium is pharmacologically inhibited, noradrenaline produces smaller contractions of arteries from NBCn1 knockout compared to wild type mice. This difference in vasoconstriction is abolished by the rho-kinase inhibitors Y-27632 and fasudil. In congruence, rhokinase-dependent phosphorylation of the myosin phosphatase targeting subunit at Thr- 850 is reduced in arteries from NBCn1 knockout mice. Based on these findings, we hypothesise that $\mathrm{NBCn} 1$ and a normal intracellular $\mathrm{pH}$ in vascular smooth muscle cells facilitate rho-kinase-dependent Ca2+-sensitivity of the contractile machinery, constriction of resistance arteries, and blood pressure responses during angiotensin II infusion. To test this hypothesis, we developed a mouse model with smooth musclespecific knockout of NBCn1 based on LoxP sites flanking exon 4 of NBCn1 and breeding with mice providing tamoxfen-inducible expression of cre recombinase under transcriptional control of the smooth muscle myosin heavy chain promoter. To evaluate functional changes, we will perform small vessel myography combined with fluorescence-based recordings of intracellular $\mathrm{pH}$ and $\mathrm{Ca} 2+$ dynamics in isolated messenteric resistance arteries. Additionally, we will challenge the mice with continuous angiotensin II infusion and study blood pressure using radiotelemetry. 
Board: $\quad 10$

Title: $\quad$ Characterization of vascular function with laser speckle imaging in vivo.

Authors: Christian Staehr and Asger Maare Sondergaard, Dmitry Postnov, Christian Aalkjaer and Vladimir Matchkov

Address:

Abstract: A novel approach of laser speckle imaging (LSI) was used to measure regional blood flow in vivo. We studied blood flow and diameter changes rat mesenteric small arteries (MSA) and regional blood flow in cerebral cortex in mice in response to whisker stimulation. By analyzing scatter shifts (i.e. fluctuating blood cells) velocity of moving objects can be determined. Contrast images of moving objects illuminated with near infrared laser light are analyzed to quantify velocity. Simultaneous measurements of vessel diameter make it possible to estimate relative changes in blood flow. This was validated with a transonic flow probe in MSA.

In intravital studies, rat MSA segments were isolated in a chamber containing a physiological salt solution. Blood flow measure by LSI was reduced by $70 \%$ in the presence of $10 \mu \mathrm{M} N \mathrm{in}$ the chamber $(n=5)$. This was consistent with flow probe measurements. Furthermore, no significant reduction in blood flow was detected in downstream arteries in the intestinal wall demonstrating the efficiency of the arcading arrangement of the intestinal circulation.

Whisker stimulation led to neuronal activity of cerebral sensory cortex of the contralateral hemisphere. Neurovascular coupling caused an increase in cerebral blood flow. A $200 \mathrm{~s}$ whisker stimulation $(5 \mathrm{~Hz}$ ) led to $9 \%$ increase in regional blood flow in sensory cortex in the contralateral hemisphere while the ipsilateral hemisphere was not significantly affected $(n=8)$. After the whisker stimulation, cerebral blood flow returned to baseline.

LSI is a convenient and relatively non-invasive approach to qualitatively access regional blood flow. 
Board: 11

Title: $\quad$ Function of TMEM16A in mouse vascular smooth muscle cells

Authors: Henrik Black Jørgensen, Dmitry Kamaev, Christian Aalkjaer, Vladimir V. Matchkov

Address: Aarhus C, Denmark

Abstract: Chloride is actively taken into smooth muscle cells (SMCs) and leaves through chloride channels causing depolarization. This depolarization will potentiate calcium influx and vasoconstriction. The TMEM16A protein was shown to be an essential part of the calcium-activated chloride channel. We aimed to study its importance for arterial contractility in different vascular beds.

We investigated the role of TMEM16A in aorta and the tail artery from SMC-specific knockout mice. Mice with LoxP sites flanking exon 7 of the TMEM16A were crossed with the inducible SMC-specific Cre mouse line and the offspring were injected with tamoxifen for 5 days. Tamoxifen-injected flox/flox mice and Cre-mice served as controls. TMEM16A expression was analyzed by Western blot. Arterial function was studied in isometric myograph. Intracellular calcium was measured using FURA-2/AM. Blood pressure was measured with telemetry.

A significant reduction in TMEM16A expression was found in tail artery $(19.4 \pm 6.6 \%$ of control, $n=5)$ and aorta $(8.7 \pm 2.6 \%$ of control, $n=4)$. Aorta knockout for TMEM16A constricted less to phenylephrine than the controls (max. constriction $76.9 \pm 10.0 \%$ of control, $n=12$ ). Surprisingly, tail artery knockout constricted stronger to phenylephrine (max. constriction $135.8 \pm 5.9 \%$ of control, $n=4$ ). Elevation of bath potassium also constricted tail arteries from TMEM16A knockouts stronger than controls, although no difference was seen for aorta. No difference in intracellular calcium changes in response to potassium or phenylephrine stimulations were seen. Blood pressure was not affected in TMEM16A knockout mice $(n=4)$.

TMEM16A function differs between different vascular beds and is not limited to the calcium-activated chloride conductance. 
Board: 12

Title: $\quad$ 4-amminopyridine enhances Kv7.4 currents, elicits smooth muscle cell hyperpolarization, decrease of [ $\mathrm{Ca} 2+] i$ and relaxation of rat mesenteric small arteries

Authors: Makhala M. Khammy ${ }^{1,2}$, Sukhan Kim², Bo H. Bentzen ${ }^{1}$, Christian Aalkjær ${ }^{1,2}$, Thomas A. Jepps ${ }^{1}$.

Address: ${ }^{1}$ Department of Biomedical Sciences, Faculty of Health and Medical Sciences, University of Copenhagen, Copenhagen, Denmark.

${ }^{2}$ Department of Biomedicine, Aarhus University, Aarhus, Denmark.

Abstract: $\quad K C N Q$-encoded channels, particularly Kv7.4 and Kv7.5 channels are known to regulate vascular tone. 4-aminopyridine is considered a broad inhibitor of $\mathrm{Kv}$ channels but is proposed to have little inhibitory effect on Kv7 family members at concentrations $<5$ $\mathrm{mM}$ (Robbins, 2001). However, the effect of 4 -aminopyridine on Kv7 channels has not been systematically studied. Thus, we investigated the pharmacological activity of 4aminopyridine on Kv7.4 and Kv7.5 channels and characterised the effect of 4aminopyridine in the vasculature.

Two-electrode voltage clamp experiments were performed on Xenopus laevis oocytes injected with CRNA encoding KCNQ4 or KCNQ5 and on freshly isolated rat mesenteric artery smooth muscle cells. The effect of 4-aminopyridine on tension, membrane potential, intracellular calcium and $\mathrm{pH}$, were assessed in rat mesenteric arteries.

4-aminopyridine increased Kv7.4 currents and shifted the voltage dependence to more negative potentials, but did not affect Kv7.5 currents in oocytes. 4-aminopyridine also enhanced native $\mathrm{K}+$ current at low millimolar concentrations. In mesenteric artery segments precontracted with noradrenaline, 4-aminopyridine hyperpolarized the membrane, decreased [Ca2+]i and caused concentration-dependent relaxation. Kv7 and BKCa channel blockade with XE991 $(1 \mu \mathrm{M})$ and iberiotoxin $(100 \mathrm{nM})$, respectively, attenuated 4-aminopyridine-mediated relaxation. Combined Kv7 and BKCa channel blockade caused further inhibition. The magnitude of the relaxation was independent of 4-aminopyridine-mediated changes in intracellular $\mathrm{pHi}$.

These data show that in noradrenaline-precontracted arteries, 4-AP can activate both Kv7.4 and BKCa channels to cause membrane hyperpolarisation and consequent decrease of $[\mathrm{Ca} 2+] \mathrm{i}$, culminating in vasorelaxation. This study cautions against using 4aminopyridine as a pan inhibitor of Kv channels at millimolar concentrations.

\section{References}

Robbins, J., 2001. KCNQ potassium channels: physiology, pathophysiology, and pharmacology. Pharmacol. Ther. 90, 1-19. 
Board: 13

Title: $\quad$ Vitamin C deficiency impairs coronary artery muscarinic receptor-mediated vasoconstriction and smooth muscle phenotypic modulation

Authors: Gry Freja Skovsted ${ }^{1 *}$, Pernille Tveden-Nyborg' ${ }^{1}$, Frederik Grell ${ }^{1}$, Karl Swärd ${ }^{2}$, Azra Alajbegovic ${ }^{2}$, Sebastian Albinsson ${ }^{2}$ and Jens Lykkesfeldt

Address: 1 Department of Veterinary and Animal Sciences, Faculty of Health and Medical Sciences, University of Copenhagen, Ridebanevej 9, 1870 Frederiksberg C, Denmark. *Correspondence: gryfreja@sund.ku.dk; Tel.: +45-3533-7705 2 Department of Experimental Medical Science, Lund University, Lund, Sweden

Abstract: Epidemiological studies have consistently found an association between vitamin $\mathrm{C}$ (vitC) deficiency and increased risk of cardiovascular disease, but the possible causal etiology has not been elucidated. In this study, we investigated the effect of vitC deficiency in guinea pigs on plasma biopterin status and vasomotor responses in coronary arteries; furthermore phenotypic modulation by vitC was studied in human coronary artery smooth muscle cells (hCASMCs). Dunkin Hartley female guinea pigs $(\mathrm{N}=32)$ were randomized to high ( $1500 \mathrm{mg} / \mathrm{kg}$ diet) or low (0 to $50 \mathrm{mg} / \mathrm{kg}$ diet) vitC for 12 weeks. Coronary artery vasomotor responses to potassium, carbachol, sodiumnitroprusside (SNP), U46619, sarafotoxin6c (S6c) and endothelin-1 (ET-1) were recorded in a wire myograph. Plasma vitC and tetrahydrobiopterin were measured by HPLC. hCASMCs were cultured in vitC deficient medium and under physiological vitC concentrations. Expression of SMC differentiation/dedifferentiation markers were investigated by qPCR.

In vivo, vitC deficient animals displayed reduced tetrahydrobiopterin $(\mathrm{p}<0.001)$. Vasoconstrictor responses to carbachol were significantly decreased in vitC deficient coronary arteries independent of their general vasoconstrictor/vasodilator capacity $(p<0.001)$. Moreover, their carbachol-induced vasodilator responses correlated with coronary artery diameter $(p<0.001)$. Responses to SNP, potassium, S6c, U46619 and ET-1 were unaffected by vitC status. In vitro, vitC deficiency delayed proliferation and significantly modulated expression of differentiation/dedifferentiation markers in hCASMCs.

Our study shows that vitC deficiency induces functional down-regulation of vasoconstrictor muscarinic receptors in guinea pig coronary arteries. Furthermore, vitC deficiency impairs hCASMC proliferation and alters expression markers of SMC phenotypic modulation. Collectively, our data support that inadequate vitC status may play a role in impaired coronary artery function and during early coronary atherosclerosis. 
Board: 14

Title: $\quad$ Effects of palmitic acid methyl ester on rat and human visceral arteries

Authors: Ning Wang*+, Artur Kuczmanski*, Galyna Dubrovska\#, Maik Gollasch\# *shared first authors; \# shared senior authors, +corresponding author

Address: Berlin, Germany

Abstract: Background: Perivascular adipose tissue (PVAT) exerts anti-contractile effects on isolated visceral arteries by release of various perivascular relaxing factors (PVRFs) and opening voltage-gated $\mathrm{K}_{+}\left(\mathrm{Kv}_{\mathrm{v}}\right)$ channels in vascular smooth muscle cells (VSMCs). Palmitic acid methyl ester (PAME) has been proposed as transferable PVRF in rat aorta. Here, we studied PVAT regulation of arterial tone of rat aorta and human mesenteric arteries with major focus on the putative role of PAME and Kv channels. Methods: Vasocontractions of isolated aortic rings from Sprague-Dawley rats and mesenteric artery rings from patients undergoing abdominal surgery were measured using wire myography. PVAT was either left intact or removed from artery rings. Vasocontractions were induced by external high $\mathrm{K}_{+}(60 \mathrm{mM})$, serotonin or phenylephrine. PAME (10 nM to $3 \mu \mathrm{M}$ ) was used as vasodilator. $K_{v}$ channels were blocked by 4 -aminopyridine, a nonspecific Kv channel inhibitor, or XE991, a Kv7 (KCNQ) channel inhibitor. PAME was measured in bathing solutions incubated with rat peri-aortic and human visceral adipose tissue. Results: We found that PVAT displayed anti-contractile effects in both rat aortas and human mesenteric arteries. The anti-contractile effects were inhibited by XE991 $(30 \mu \mathrm{M})$. PAME (EC $5014.2 \mu \mathrm{M})$ was capable to produce relaxations of PVATremoved rat aortas. These effects were abolished by XE991 $(30 \mu \mathrm{M})$, but not 4aminopyridine $(2 \mathrm{mM})$ or NDGA $(10 \mu \mathrm{M})$, a lipoxygenases inhibitor. Although the cytochrome P450 epoxygenase inhibitor 17-octadecynoic acid (ODYA $10 \mu \mathrm{M})$ slightly inhibited PAME relaxations in rat aorta, the soluble epoxide hydrolase inhibitor 12-(3adamantan-1-ylureido)-dodecanoic acid (AUDA $10 \mu \mathrm{M}$ ) did not increase PAME relaxations. PAME up to $3 \mu \mathrm{M}$ failed to induce relaxations of PVAT-removed human mesenteric arteries. Endogenously released PAME levels were detected in bathing solutions of both rat peri-aortic and human visceral adipose tissues. Serotonin did increase PAME release from rat peri-aortic adipose tissue, but not from human visceral adipose tissue. Conclusions: Our data suggest that Kv7 (KCNQ) channels are involved in PVAT regulation of arterial tone in both rat aorta and human visceral arteries. PAME displays properties of PVRF in rat aorta, but not in human mesenteric arteries, where it may contribute to PVAT regulation of arterial tone, independently of metabolism of endogenous lipid epoxides. 
Board: 15

Title: Overexpression of SIRT1 in vascular endothelial cells prevents premature vascular and metabolic ageing in Per2 mutant mice

Authors: Andy WC Man, Ellie Q Liu, Yumeng Guo, Cuiting Luo, Aimin Xu, Paul M Vanhoutte, Yu Wang*

Address: ${ }^{1}$ State Key Laboratory of Pharmaceutical Biotechnology and Department of Pharmacology and Pharmacy, The University of Hong Kong, Hong Kong

Abstract: Background- SIRT1 is a longevity regulator implicated in the regulation of circadian rhythmicity, which facilitates the organisms to adapt to the external environment. PER2 is a member of the Period family of circadian regulators participating in the transcription-translational feedback loop of clock-control mechanisms. The present study was designed to evaluate the protective effects of SIRT1 in vascular endothelial cells on Per2 mutation-induced vascular and metabolic abnormalities.

Methods and Results-Wild type (WT) and PER2 mutant (Per2 ${ }^{\mathrm{Brdm} 2}$ ) mice without or with human SIRT1 selectively overexpressed in vascular endothelial cells (Per2 ${ }^{\mathrm{Brdm}}{ }^{\mathrm{ECC}}$ SIRT1) were used in the present study. Mice were housed under 12-hour light/dark cycle and a controlled temperature conditions with free access to water and chow. Radiotelemetry transmitters were implanted for monitoring arterial blood pressures. Compared to WT mice, Per2 ${ }^{\text {Brdm2 }}$ mice exhibited a loss of dipping in the diurnal blood pressures at the age of eight-weeks and hypertension at the age of 24-weeks, both of which were prevented by SIRT1 overexpression in endothelial cells. The premature metabolic ageing phenotype caused by Per 2 mutation, including decreased skeletal muscle mass and increased fat mass as well as altered insulin sensitivity, was also prevented by endothelial overexpression of SIRT1. Preliminary metabolomics analyses indicated that endothelial SIRT1 elicits the anti-vascular and metabolic ageing activities via modulating amino acid metabolism in skeletal muscle.

Conclusion-Endothelial SIRT1 represents a promising target for anti-ageing therapies in both vascular and metabolic diseases.

Reference

1. Zu, Y., Liu, L., Lee, M. Y., Xu, C., Liang, Y., Man, R. Y., ... \& Wang, Y. (2010). SIRT1 promotes proliferation and prevents senescence through targeting LKB1 in primary porcine aortic endothelial cells. Circulation research, 106(8), 13841393.

2. Chang, H. C., \& Guarente, L. (2013). SIRT1 mediates central circadian control in the SCN by a mechanism that decays with aging. Cell, 153(7), 1448-1460.

3. Asher, G., Gatfield, D., Stratmann, M., Reinke, H., Dibner, C., Kreppel, F., ... \& Schibler, U. (2008). SIRT1 regulates circadian clock gene expression through PER2 deacetylation. Cell, 134(2), 317-328.

4. Bellet, M. M., Orozco-Solis, R., Sahar, S., Eckel-Mahan, K., \& Sassone-Corsi, P. (2011, January). The time of metabolism: NAD+, SIRT1, and the circadian clock. In Cold Spring Harbor symposia on quantitative biology (Vol. 76, pp. 31-38). Cold Spring Harbor Laboratory Press. 
Board: 16

Title: $\quad$ A novel in vitro approach to study steady-state conduction of vasomotor responses in small arteries

Authors: Teresa Palao, Angela van Weert, Anne de Leeuw, Judith de Vos, Erik N.T.P. Bakker, Ed van Bavel

Address: Department of Biomedical Engineering and Physics, Academic Medical Center, Amsterdam, The Netherlands

Abstract: Local vasoactive responses of resistance arteries in vascular networks need to be coordinated in order to effectively regulate tissue perfusion. Conducted responses may form the base for such coordination. As a first step for understanding network behaviour, we studied conduction along single resistance vessel segments.

Studies on conduction of tone so far focused on the conducted vasodilatory response to acute local stimuli. However, steady-state coordination of tone is also required to maintain a stable diameter along the vessel. We hypothesized that steady-state stimulation of part of a vessel modulates tone along the full vessel length.

To study this, we designed a new in vitro set up consisting of a modification of the pressure myograph, where the superfusion chamber is divided into two compartments by a septum halfway along the vessel. The setup was first tested to ensure vascular viability and absence of drug diffusion to non- stimulated parts of the vessel. Then steady-state responses to different vascular constrictors and dilators were tested. Phenylephrine induced a small conducted contractile response in the non-stimulated site only at high concentrations. Contractions induced in the local segment were slightly smaller that contractions in the fully stimulated vessel. Potassium did not induce conducted responses up to $100 \mathrm{mM}$. Conducted vasodilatory responses to acetylcholine were clearly present, showing up to $50 \%$ dilation on the non-stimulated part of the vessel.

In conclusion, we developed a new setup for studying steady-state conduction of tone along resistance vessel segments. The results indicate that local vasoconstrictors have very limited distal effects under the current conditions. However, the steady state conduction of endothelium-dependent vasodilation is relatively strong.

Supported by FP7 Marie Curie grant 606998. 
Board: 17

Title: $\quad$ Pulse Pressure affects Remodeling of Small Arteries in Organoid Culture

Authors: Anne de Leeuw, Judith Vos, Erik Bakker, Peter Hordijk, Ed van Bavel

Address: Dept. of Biomedical Engineering and Physics, Academic Medical Center and Dept. of Physiology, Free University Medical Center, Amsterdam, The Netherlands

Abstract: Cannulated vessels form one of the core in vitro approaches for resistance artery research. This technique has been extended to the study of remodeling of pressurized vessels culture. We previously demonstrated inward remodeling in cremaster muscle arteries in the presence but not the absence of pressure (Bakker et al., J Vasc Res. 2004;41:174-82). Yet, while arterial pressure is pulsatile, this and other studies were performed under a constant pressure load. We tested whether the maintenance of a normal pulsatile pressure affects remodeling of resistance vessels.

Rat mesenteric small arteries were kept in culture for 3 days at either constant pressure $(60 \mathrm{mmHg})$, or a pulsatile pressure ranging between $40-80 \mathrm{mmHg}$ at $\sim 300$ BMP. Extravascular fluid was Leibovitz culture medium; the luminal fluid contained medium with $10 \%$ fetal calf serum. Vascular reactivity was tested at the start and end the culture period. Remodeling was determined from the change in pressure-diameter relations under full vasodilation induced with papavarin. Both inner and outer diameter were recorded to detect possible hypertrophy.

Both groups showed significant remodeling after 3 days of incubation $(p<0.001)$. Wall surface area in the constant pressure group increased, indicating hypertrophic remodeling. In contrast, the group incubated under pulsatile pressure maintained a constant wall surface area. Expression of the contractile protein $\alpha$-Smooth Muscle Actin was increased, demonstrated with RT-PCR. Immune staining further indicated that this increase was localized in the adventitia of non-pulsatile arteries, indicating that the hypertrophic remodeling was characterized by myofibroblast activation in this vascular layer.

In conclusion, resistance artery remodeling is affected by pulse pressure. These data plea for application of pulsatile pressures in acute and long-term studies on cannulated resistance vessels.

Support: Amsterdam Cardiovascular Science grant. 
Board: 18

Title: Blood pressure response to norepinephrine and epinephrine after sympathectomy of spontaneously hypertensive rat

Authors: Vavř́nová $\mathrm{A}^{1,2}$, Behuliak $\mathrm{M}^{1}$, Bencze $\mathrm{M}^{1,2}$, Zicha $\mathrm{J}^{1}$

Address: ${ }^{1}$ Department of Experimental Hypertension, Institute of Physiology, The Czech Academy of Sciences, Prague, Czech Republic

${ }^{2}$ Charles University in Prague, Faculty of Science, Prague, Czech Republic

Abstract: Sympathetic innervation of resistance arteries and sympathoadrenal system play an important role in development of high blood pressure in spontaneously hypertensive rat (SHR). We performed chemical sympathectomy (SYMPX) on 20-week-old SHR and normotensive Wistar-Kyoto (WKY) rats by daily intraperitoneal administration of guanethidine (30 mg/kg per day) for two weeks. SYMPX was confirmed by visualization of catecholamines in femoral artery by sucrose-potassium phosphate-glyoxylic acid (SPG) solution. Blood pressure was measured in conscious animals 24 hours after cannulation of carotid artery. Plasma levels of norepinephrine and epinephrine were measured in intact control and sympathectomised rats. Control SHR had higher basal mean arterial pressure (MAP) and heart rate (HR) than WKY rats. SYMPX decreased both parameters, the strain difference being preserved. The effect of acute intravenous administration of norepinephrine or epinephrine on MAP and HR did not differ between control SHR and WKY rats. After SYMPX, MAP response to norepinephrine or epinephrine was shifted to the left and increased in both strains, the effects being augmented in SHR. Plasma level of catecholamines did not differ between control SHR and WKY rats. Plasma norepinephrine decreased after SYMPX in both strains, while plasma epinephrine increased after SYMPX, the effect being more pronounced in WKY. MAP decrease after sympathectomy of SHR and WKY rats seems to be counteracted by increased sensitivity of arteries to catecholamines and by increased plasma level of epinephrine. It would be interesting to study molecular basis of the compensatory changes in arteries and in adrenal medulla of sympathectomised rats.

(GACR16-10349Y, GAUK1071416) 
Board: $\quad 19$

Title: $\quad$ Postnatal maturation attenuates the impact of Kv7 channels on smooth muscle membrane potential and contractility in rat arteries

Authors: A.A. Shvetsova ${ }^{1,2}$, D.G. Gaynullina ${ }^{1,2}$, R. Schubert ${ }^{3}$, O.S. Tarasova ${ }^{1,2}$.

Address: $\quad{ }^{1}$ SRC RF Institute for Biomedical Problems RAS, Moscow, Russia

${ }^{2}$ Faculty of Biology, Lomonosov Moscow State University, Moscow, Russia

${ }^{3}$ Heidelberg University, Medical Faculty Mannheim, Germany

Abstract: Objectives. Vascular smooth muscle Kv7 potassium channels contribute considerably to agonist and pressure-induced vasoconstriction and to vasodilation induced by various substances. Notably, potassium channel function has been shown to be altered during postnatal ontogenesis. However, the role of Kv7 channels to this process has not been studied yet. We tested the hypothesis that the impact of Kv7 channels in counteracting vasocontractile responses changes with maturation.

Materials and Methods. All experiments were performed on endothelium-denuded saphenous arteries from young (10-15-day) and adult (2-3-month) male rats. Isometric force and membrane potential (MP) were recorded simultaneously using wire myography and the sharp microelectrode technique. The functional role of Kv7 channels was studied using the specific inhibitor XE991 (3 microM). Kv7 channel subunit expression was determined by qPCR.

Results. In adult arteries XE991 caused a small depolarization $(9 \mathrm{mV})$, not associated with tone development. In contrast, in young arteries strong depolarization $(25 \mathrm{mV})$ and basal tone development were observed in response to XE991. Moreover, XE991induced a potentiation of the depolarization and the contractile response evoked by the $\alpha 1$-adrenoceptor agonist methoxamine. This effect was more prominent in young compared to adult arteries. Resting MP levels did not differ between the two age groups. In addition, a higher abundance of Kcnq1, Kcnq2, Kcne2, Kcne3 and Kcne4 mRNA was observed in arteries of young rats.

Conclusions. The contribution of Kv7 channels to the regulation of contractile responses of rat saphenous arteries is stronger in early postnatal ontogenesis.

Supported by RFBR (grant N16-04-01395) and DAAD (Short-Term Grants, 2016). 
Board: $\quad 20$

Title: Characterization of an intimal hyperplasia model in mice with vascular risk factors

Authors: Marycarmen Arévalo-Martínez, Esperanza Alonso, Irene Cózar, José R López-López, M. Teresa Pérez-García and Pilar Cidad

Address: Departamento de Bioquímica y Biología Molecular y Fisiología, Facultad de Medicina and IBGM, Universidad de Valladolid and CSIC, Valladolid, SPAIN

Abstract: Vascular smooth muscle cells (VSMCs) of the vessel wall are normally quiescent, contractile cells. However, mechanical or chemical injury promotes dedifferentiation of the VSMCs, triggering a phenotypic modulation (PM) directed to neointima formation. This leads to restenosis, which is the main cause of failure of vascular interventions. Besides, vascular surgery outcome depends on underlying diseases, and patients with associated vascular risk factors (VRF) show accelerated restenosis and worse prognosis. We have previously found that PM associates with changes in the expression of the voltage-dependent potassium channel Kv1.3 and Kv1.5. Kv1.3 blockers inhibit proliferation and migration in cultured human VSMCs and intimal hyperplasia in a mice model of endoluminal lesion.

Here, we propose to explore the efficacy of Kv1.3 blockers preventing PM in mice showing VRF. Intimal hyperplasia was generated by carotid artery ligation in hypertensive mice with high fat diet (BPH/HFD). Under these conditions, BPH mice developed metabolic syndrome. Hystomorphometric analysis, qPCR and immunohistochemistry are used to measure vascular remodeling and changes in expression of VSMCs markers. HFD per se produce remodeling of the vessel wall by augmenting VSMCs contraction. Moreover, there is an increased expression of Kv1.3 mRNA in HFD vessels, which suggest that Kv1.3 blockers could be more effective preventing PM in these animals. Finally, carotid ligation of HFD animals induced a larger remodeling with increased stenosis.

We conclude that BPH/HFD could represent a good model to study the influence of underlying VRF on the prevention and treatment of restenosis in high-risk patients.

Supported by grant BFU2016-75360-R (MINECO) and by a UVa-Santander fellowship to MAM. 
Board: 21

Title: $\quad$ MARCKS regulates vascular contractility

Authors: Jahan KS, Shi J, Greenwood IA, Albert AP

Address: Vascular Biology Research Centre, St George's University of London, London, United Kingdom

Abstract: The present work investigates the role of Myristoylated alanine-rich C-Kinase (MARCKS) in vascular smooth muscle cells (VSMCs).

Experiments were carried out using freshly isolated VSMCs and tissue lysates from rat and mice mesenteric arteries alongside western blot, immunocytochemical, coimmunoprecipitation, dot-blot and patch clamp techniques.

MARCKS was predominantly expressed at the plasma membrane of VSMCs. The cellpermeable MARCKS inhibitor, MANS peptide, evoked concentration-dependent increases in vascular contractility that were abolished by VDCC blockers. Knockdown of MARCKS significantly reduced contractions evoked by MANS peptide. In un-stimulated tissue, MARCKS interacted with the L-type Ca2+ channel protein, CaV1.2, and PIP2. MANS peptide released PIP2 from MARCKS and increased interactions between CaV1.2 and PIP2. MANS peptide induced an increase in the peak amplitude and a negative shift in activation and inactivation of whole-cell Ca2 + currents. Depletion of PIP2, with wortmannin, abolished excitatory effects of MANS peptide on whole-cell VDCC activity.

This is the first study to demonstrate that MARCKS is involved in regulating vascular contractility. We propose that in un-stimulated vessels, MARCKS prevents opening of Ltype Ca2+ channels by acting as a PIP2 buffer, but following MARCKS inhibition PIP2 is released leading to channel gating and contractility. 
Board: 22

Title: Differential effects of acetylcholine and bradykinin on eNOS and COX mediated skeletal muscle arterial dilatation

Authors: Asmaa Raees ${ }^{1}$, David Abraham ${ }^{2}$, Mashael Al-Jaber ${ }^{1}$, Aysha A Bakhamis ${ }^{1}$, Vidya Mohamed-Ali ${ }^{1}$, Muhammad Al-Sayrafi ${ }^{1}$, Nelson N Orie ${ }^{1}$.

Address: $\quad{ }^{1}$ Life Sciences Research Division, Anti-Doping Lab Qatar, ${ }^{2}$ Division of Medicine, University College London.

Abstract: Nitric oxide and prostacyclin are endothelium-derived vasodilators in most vascular beds. Specifically, in arteries from skeletal muscle, there are inconsistencies in the reported effects of blocking either endothelial nitric oxide synthase (eNOS) or the cyclooxygenase (COX) pathways. Functional redundancy between both pathways might explain these observed inconsistencies. The present study investigated the effects of two endothelium-dependent dilators, acetylcholine (Ach) and bradykinin (BK) to elucidate the mechanism(s) of eNOS and COX contributions to vasodilation in these arteries.

Small arteries $(264 \pm 14 \mu \mathrm{m}, \mathrm{n}=26)$ were isolated from ovine abdominal muscle, obtained from the slaughter house in Doha, Qatar. Relaxation to Ach and BK were studied by wire myography in the absence or presence of $\mathrm{N} \omega$-Nitro-L-arginine methyl ester (L-NAME, $100 \mu \mathrm{M}$, NOS inhibitor), indomethacin (10 $\mu \mathrm{M}$, COX inhibitor) and/or 30 $\mathrm{mM} \mathrm{KCl}$ (blocks hyperpolarization).

The arteries were relaxed by both Ach and BK with $\log \mathrm{IC}_{50}$ of $-7.57 \pm 0.10$ and $9.44 \pm 0.20$ respectively, representing significantly greater sensitivity to $B K(p<0.0001)$. Curves for both agonists were shifted to the right by L-NAME, indomethacin and 30 $\mathrm{mM} \mathrm{KCl}$. The shift of Ach curves by L-NAME or indomethacin was enhanced by previous treatment with indomethacin or L-NAME, respectively. Conversely, BK curves were independently shifted to the right by either L-NAME $(p<0.001)$ or indomethacin $(p<0.01)$, with no apparent influence of previous treatment.

There are unique differences in the involvement of eNOS and COX in Ach and BK relaxation of ovine skeletal muscle arteries, with redundancy between the pathways specific to Ach, but not to BK. 
Board: 23

Title: $\quad$ The effects of age on the biomechanical properties of cerebral arteries

Authors: Torrens C, Ewbank FG

Address: Faculty of Medicine, University of Southampton, University Road, Southampton SO17 $1 \mathrm{BJ}$.

Abstract: Background: While the age- related properties of large elastic arteries have been well characterised, there has been much conjecture concerning the effects of age on the biomechanical properties of resistance- sized cerebral arteries.

Aims: The aim of this study was to characterise the effects of age on the biomechanical properties of cerebral arteries.

Methods: The middle cerebral artery of young (14- 16 weeks old) and old (56- 58 weeks old) male Wistar rats were used in this study. Segments of artery were mounted on a wire myograph $(n=5)$. Passive properties were assessed by multiple incremental stretches of $0.05 \mathrm{~mm}$ every 2 minutes. For each incremental stretch, the peak force, plateau force, increase in force and difference in force can be measured. Separate segments were fixed and stained using Miller's method $(n=4)$. Wall thickness and composition were analysed using ImageJ software.

Results: Statistical analysis showed a significant reduction in the tension measured at all points in old arteries $(p<0.05)$. However, the percentage decrease in tension for each incremental stretch was not significantly different $(p>0.05)$. There was no significant difference in arterial compliance or internal circumference measured at 100 $\mathrm{mmHg}(p>0.05)$. Histological analysis showed no significant effect of age on wall thickness or arterial composition ( $p>0.05$ ).

Conclusion: Overall, these results suggest that cerebral artery stiffness is reduced with age. Moreover, this reduction in arterial stiffness is not associated with changes in luminal diameter, wall thickness, compliance or composition. A reduction in wall stress in response to age may suggest a loss of the Bayliss effect and, therefore, cerebrovascular autoregulation. Furthermore, absent changes in arterial composition has led to the suggestion that dysfunctional vascular smooth muscle cells may result in these biomechanical changes. 
Board: 24

Title: Identification of a $\mathrm{G}$ protein $\beta \gamma$ subunit dependent vasorelaxation pathway involving Kv7 channels

Authors: Jennifer B Stott, Vincenzo Barrese, Malavika Suresh, Shirou Masoodi and Iain A Greenwood

Address: Vascular Research Group, Institute of Molecular and Clinical Sciences, St George's University of London, London, UK

Abstract: Kv7 channels are important regulators of vascular responsiveness, including relaxations induced by G-protein coupled receptor (GPCR) stimulation. Activation of GPCRs promotes dissociation of $\mathrm{Ga}$ from $\mathrm{G} \beta \gamma$ subunits to activate numerous intracellular signalling events. Whilst the pathways downstream of Gas which promote vasorelaxation are well characterised, the role of $G \beta \gamma$ subunit signalling in these events is not clear. In addition, recently native vascular Kv7 channels were shown to be regulated by $\mathrm{G} \beta \gamma$ subunits. Here, we investigate the role of $\mathrm{G} \beta \gamma$ signalling in vasorelaxations via Kv7 channels in the vasculature.

Proximity ligation assay showed a higher basal interaction of Kv7.4-G $\beta$ in renal than mesenteric arteries (RA and MA, respectively). Application of MSIRK (promotes disassociation of $G \beta \gamma$, without stimulation of $\mathrm{Ga}$ ) produced relaxations of both MA and RA, which were Kv7 dependent. Stimulation of isolated myocytes with mSIRK increased Kv7.4-G $\beta$ puncta in MA but not RA. Dose dependent relaxations to isoprenaline in MA and RA are Kv7 dependent. Relaxations to isoprenaline in rat renal, but not mesenteric arteries, were impaired in the presence of $G \beta \gamma$ inhibitors. In MA, but not the RA, isoprenaline relaxations are mediated by the exchange protein activated by cyclic AMP (EPAC) and increase the association of Kv7.4 with the small G protein Rap1a. Gallein treatment did not affect EPAC dependent relaxations, or the resultant association of Kv7.4-Rap1a. This suggests that $G \beta \gamma$ are required for some intracellular signalling effects on KV7 channels, but not EPAC mediated ones. 
Board: 25

Title: The tryptophan metabolite kynurenine induces vasorelaxation in systemic maternal arteries: a new target for therapeutic intervention?

Authors: Stephanie A Worton, Mark Wareing, Susan L Greenwood, Jenny E Myers

Address: Maternal and Fetal Health Research Centre, University of Manchester, $5^{\text {th }}$ Floor, St Mary's Hospital, Hathersage Road, Manchester, UK

Abstract: Kynurenine (Kyn) relaxes blood vessels and reduces blood pressure in animal models via guanylate cyclase (GC) and adenylate cyclase (AC)1. In a single small human study, Kyn relaxed omental arteries and this was attenuated by Linopirdine, an inhibitor of type-7 voltage-gated potassium (Kv7) channels2. The Kyn pathway of tryptophan metabolism is less active in $\mathrm{PE}$, a condition characterised by increased systemic arterial tone. This study aimed to determine if Kyn can regulate vascular tone in systemic resistance arteries in pregnancy.

Methods: Omental resistance arteries ( $<500 u M)$ obtained from uncomplicated, term pregnancies were assessed by wire myography. Dose-response curves to Kyn (0.05$3 \mathrm{mM}), \mathrm{Kyn}+$ inhibitor or vehicle diluent were constructed for arteries pre-constricted with U-46619 (EC80). Inhibitors were as follows: (i) soluble GC; ODQ 10 $\mu \mathrm{M}, 30 \mathrm{mins}$

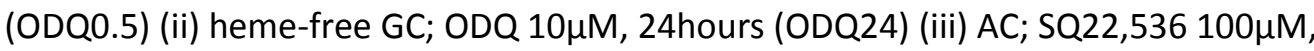
30 mins (iv) Kv7; Linopirdine $10 \mu \mathrm{M}, 30$ mins. Responses were compared by 2-way repeated-measures ANOVA with Sidak's post-tests $(p<0.05)$.

Results: Kyn induced relaxation of pre-constricted omental arteries $(\mathrm{N}=25, \mathrm{p}<0.0001$; relaxation $41.7+/-5.40 \%$ vs. $78.5+/-3.10 \%$ ). Treatment with ODQ0.5, ODQ24, SQ22,536 or Linopirdine had no effect on Kyn-induced relaxation ( $N=5-11$ each inhibitor, $\mathrm{p}=\mathrm{ns})$.

Discussion: Kyn causes substantial and consistent vasorelaxation of omental resistance arteries from pregnant women, supporting our hypothesis that restoring Kyn pathway activity in pre-eclampsia could be a novel therapeutic target to attenuate maternal features of the disease. In omental arteries from pregnant women, Kyn-induced relaxation was not mediated via GC, AC or Kv7 channels, suggesting a novel mechanism of Kyn activity.

[1] Wang et al. Nat Med. 2010;3:279-85 [2] Sakakibara et al. J Pharmacol Sci. 2015;129:31-37 
Board: 26

Title: $\quad$ Microvascular tone of skeletal muscle resistance arteries: The regulatory role of perivascular adipose tissue and insulin

Authors: Ben Clark $^{1}$, Sarah Withers ${ }^{1,2}$, Anthony Heagerty ${ }^{1}$

Address: ${ }^{1}$ Division of Cardiovascular Sciences, The University of Manchester, United Kingdom ${ }^{2} \mathrm{BRC}$, Environment and Life Sciences, The University of Salford, United Kingdom

Abstract: Objective: Resistance arteries significantly contribute to peripheral vascular resistance, and also determine insulin-sensitivity by controlling blood flow to skeletal muscle. Increased microvascular tone therefore facilitates the development of hypertension and insulin-resistance. Surrounding most blood vessels, perivascular adipose tissue (PVAT) exerts a vasorelaxant 'anti-contractile' effect. Few studies on skeletal muscle resistance arteries have investigated this vasorelaxant effect and the role of insulin. We aimed to address this through establishing a novel in vitro model.

Design and Method: Vascular contractility of arterial segments, with (+) or without (-) PVAT, from hindlimb skeletal muscle resistance arteries of healthy male Wistar rats was assessed by wire myography. Viable vessels achieved tensions $0.3 \mathrm{mN} / \mathrm{mm}$ when challenged with $60 \mathrm{mM}$ high-K+ physiological saline solution (KPSS). Arterial segments were subsequently pre-constricted with noradrenaline $(30 \mu \mathrm{M})$ and endothelial function was confirmed by vasodilation ( $25 \%$ reduction of noradrenaline-induced tension) in response to carbachol $(30 \mu \mathrm{M}, \sim 10 \mathrm{~min})$. Vessels were challenged with either serotonin or noradrenaline (-8 to $-4.5 \log 10 \mathrm{M})$. The effects of insulin $(2 \mathrm{nM}, 30 \mathrm{~min}$ preincubation) were investigated on the noradrenaline-induced dose-response. Responses were normalised to post-dose-response KPSS-induced constrictions. Statistical analyses were performed via two-way ANOVA with Bonferroni post-hoc analysis.

Results: PVAT exerted an endothelium-independent anti-contractile effect in response to serotonin (-PVAT: $n=8 ;+$ PVAT: $n=8 ; P<0.0001$ ), which was potentiated by endothelium (-Endothelium: $n=8 ;+$ Endothelium: $n=3 ; P<0.0001$ ). Contrastingly, endothelium but not PVAT exerted anti-contractility in response to noradrenaline (Endothelium $n=8,+$ Endothelium $n=10, P<0.0001$ ). Following insulin pre-incubation, noradrenaline induced either PVAT-mediated anti-contractility or pro-contractility depending upon the absence (Noradrenaline: $n=8$; Insulin: $n=4 ; P=0.009$ ) or presence (Noradrenaline: $n=10$; Insulin: $n=2 ; P=0.0007$ ) of endothelium.

Conclusions: Serotonergic and adrenergic stimulation elicit anti-contractile responses through PVAT and the endothelium, respectively. Consistent with previous studies, insulin acts as both a vasodilator and vasoconstrictor. This model should allow for obesity-associated microvascular dysfunction, in which PVAT and endothelial function are impaired, to be characterised in skeletal muscle resistance arteries. 
Board: $\quad 27$

Title: The Effects of Cyclosporin A and Other Small Molecular Inhibitors of Cyclophilin D on Hypoxia in Perivascular Adipose Tissue

Authors: $\quad$ Robert G Aldous ${ }^{1}$, Sophie N Saxton ${ }^{1}$, Lu-Yun Lian ${ }^{2}$, Michael Rogers ${ }^{3}$, Neil Berry ${ }^{3}$, Paul $\mathrm{O}^{\prime}$ Neill $^{3}$ \& Anthony M Heagerty ${ }^{1}$.

Address: $\quad{ }^{1}$ Division of Cardiovascular Sciences, University of Manchester ${ }^{2}$ Institute of Integrative Biology, University of Liverpool

${ }^{3}$ Department of Chemistry, University of Liverpool

Abstract: Hypertension affects approximately one third of the UK population, and the incident of hypertension is significantly increased in obesity. In health, perivascular adipose tissue (PVAT) surrounds most blood vessels and exerts an anti-contractile effect on arteries through the release of adipose-derived relaxing factors. In obesity, adipose depots become hypoxic; inducing oxidative stress and an inflammatory response, which may be contributing to the development of hypertension. In response to hypoxia, cyclophilin $\mathrm{D}(\mathrm{CypD})$ regulates mitochondrial pore opening which will ultimately lead to cell death. Therefore inhibition of CypD may be useful in preventing adipocyte dysfunction in obesity. This study examined the influence of CypD inhibition on vascular contractility and the PVAT anti-contractile effect under normoxic and hypoxic conditions. Contractility of mouse mesenteric arteries ( \pm PVAT) was tested using a noradrenaline concentration response in the presence and absence of three CypD inhibitors; cyclosporin A (CSA), 3C117 and 3CD02. PVAT exerted an anti-contractile effect on the mesenteric arteries, and the CypD inhibitors had no effect on vascular contractility or PVAT function. To examine the effects of hypoxia, organ baths were infused 95\% N2 / 5\% CO2 for 2.5 hours. The PVAT anti-contractile effect was abolished by hypoxia; however this loss of function could be prevented using incubation with all three CypD inhibitors. In conclusion, this study indicates that hypoxia may play a vital role in the loss of PVAT function in obesity, and inhibition of CypD presents a potential pharmacological target in the prevention of adipose tissue dysfunction, therefore preventing the development of hypertension. 


\section{Board: $\quad 28$}

Title: $\quad$ Smooth muscle or endothelial cell knockout of KIR2.1 does not inhibit K+-induced vasodilation in murine superior epigastric arteries.

Authors: William F. Jackson, Nathan R. Tykocki and Mark T. Nelson

Address:

Abstract: Endothelial cell (EC) and smooth muscle cell (SMC) inward rectifier K+ channels (KIR) mediate resistance artery dilation to increases in extracellular $\mathrm{K}+([\mathrm{K}+] \mathrm{o})$ and serve as amplifiers of hyperpolarization induced by other channels/transporters. KIR2.1 mediates these functions in cerebral SMCs and in mesenteric ECs. However, the role of KIR2.1 has not been examined in other vessels. We tested the hypothesis that both EC and SMC KIR2.1 mediate [K+]o-induced vasodilation in murine superior epigastric arteries (SEAs). SEAs were isolated from male C57BL6, EC-specific KIR2.1 knockout (ECKO), and tamoxifen-inducible SMC-specific KIR2.1 knockout (SMCKO) mice and studied by pressure myography. Raising [K+]o from 5 to 8 or $15 \mathrm{mM}$ resulted in $44 \pm 7 \%$ and $68 \pm 10 \%$ dilation, respectively in C57BL6 SEAs ( $n=6-10)$. Similar dilations occurred in ECKO SEAs: $8 \mathrm{mM} \mathrm{K+}=32 \pm 10 \%$ dilation and $15 \mathrm{mM} \mathrm{K}+=82 \pm 6 \%$ dilation $(n=5 ; p>0.05$ vs. C57BL6). [K+]o-induced vasodilation also was not inhibited in SMCKO SEAs: $8 \mathrm{mM} \mathrm{K+=}$ $26 \pm 6 \%$ dilation and $15 \mathrm{mM} \mathrm{K}+=88 \pm 7 \%$ dilation ( $n=4 ; p>0.05$ vs C57BL6). In all 3 models, $100 \mu \mathrm{M} \mathrm{Ba} 2+$ inhibited [K+]o induced vasodilation, substantiating roles for KIR. Western blots of C57BL6 whole-SEA lysates revealed protein for both KIR2.1 and KIR2.2. Immunofluorescent labeling of isolated ECs and SMCs revealed that ECs expressed exclusively KIR2.1, whereas SMCs expressed both KIR2 isoforms. Our data suggest that, in murine SEAs, EC KIR2.1 does not participate in [K+]o-induced vasodilation, and that a SMC KIR other than KIR2.1 (possibly KIR2.2?) may compensate for SMC-specific loss of KIR2.1.

Supported by P01

HL070687 (WFJ); K01-DK103840 (NRT); R01-HL121706, R01-HL131181 (MTN). 


\section{Tuesday Poster Session}

\section{The Wilson Suite}

\section{$4 \mathrm{pm}$ to $6 \mathrm{pm}$}

Abstracts in board number order

Please ensure you display your poster from $3.30 \mathrm{pm}$ to $6 \mathrm{pm}$.

Moderation will be ongoing during the poster session. Please ensure you stay with your poster during this time

Any posters left at the end of the day will be removed. 
Board: 1

Title: Increased functional expression of P2Y6 receptors in mesenteric arteries from hypertensive mice.

Authors: Inés Alvarez-Miguel, Pilar Cidad, Esperanza Alonso, Tania Arranz, José R. López-López and M Teresa Pérez-García

Address: Departamento de Bioquímica y Biología Molecular y Fisiología, Facultad de Medicina and IBGM, Universidad de Valladolid and CSIC, Valladolid, SPAIN

Abstract: In vascular smooth muscle cells (VSMCs), the membrane depolarization caused by activation of DAG signaling pathway leads to VOCCs channels opening, [Ca2+]i increase and contraction. We have previously shown an increased functional expression of a member of the TRPC family of non-selective cation channels, namely TRPC3, in mesenteric VSMC of hypertensive mice which contributes to the increased vascular tone by favoring depolarization (Alvarez-Miguel et al. 2017). Changes in the functional expression of G-protein coupled receptors (GPCRs) could alter DAG-dependent activation of TRPC3 contributing to the altered vascular tone. In this context, we explored the molecular constituents of this signaling pathway and their contribution to the hypertensive phenotype.

We used mesenteric arteries and isolated VSMCs from a mice model of essential hypertension (BPH) with its normotensive control (BPN). Pressure myography in endothelial-denuded arteries showed vasoconstriction in response to ATP and UTP.

ATP responses were similar in BPN and BPH arteries and were sensitive to the P2X1 blocker $\alpha-\beta$-methylene-ATP. However, UTP- and UDP-induced vasoconstrictions were insensitive to $\alpha-\beta$-methylene-ATP and significantly larger in BPH arteries. Both the pharmacological profile of these responses and the $\mathrm{qPCR}$ expression studies indicated an increased expression of $\mathrm{P} 2 \mathrm{Y} 6$ receptors in $\mathrm{BPH}$ arteries. A fraction of this vasoconstriction was inhibited by niflumic acid and bumetanide, pointing to the involvement of $\mathrm{Ca}_{2}+$-activated $\mathrm{Cl}$ - $(\mathrm{ClCa})$ channels and the $\mathrm{Na}_{+}-\mathrm{K}_{+}-2 \mathrm{Cl}$ - cotransporter, NKCC1, which were confirmed with electrophysiological studies in isolated VSMCs. We propose a role for the augmented activity of the P2Y6 receptor pathway in the increased vascular tone in hypertension.

Funding. Supported by grants BFU2016-75360-R (MINECO) and by a Junta de Castilla y León and Fondo Social Europeo (FSE) fellowship to IAM. 


\section{Board: 2}

Title: $\quad \mathrm{K}_{\mathrm{IR}}$ channels are required for modulation of myogenic tone by $\mathrm{K}_{\mathrm{Ca}}$ channel activators

Authors: $\quad$ R. Wei ${ }^{1}, \mathrm{PM} . \mathrm{Kerr}^{2}$, F. Plane ${ }^{1}$

Address: $\quad{ }^{1}$ Department of Pharmacology, University of Alberta, Edmonton, AB., Canada,

${ }^{2}$ Department of Nursing Science, MacEwan University, Edmonton, AB., Canada

Abstract: Background: Opening of endothelial small $\left(\mathrm{SK}_{\mathrm{Ca}}\right)$ and intermediate $\left(\mathrm{IK}_{\mathrm{Ca}}\right)$ conductance $\mathrm{Ca}^{2+-}$ activated $\mathrm{K}^{+}$channels leads to hyperpolarization which spreads to surrounding smooth cell muscle cells via gap junctions to inhibit contraction. The activity of inward rectifier $\mathrm{K}^{+}\left(\mathrm{K}_{\mathrm{IR}}\right)$ channels increases with membrane hyperpolarization. Thus, we have investigated whether pharmacological activators of $\mathrm{SK}_{\mathrm{Ca}}$ and IKCa channels engage KIR channels to modulate myogenic reactivity in resistance arteries.

Methods: 3-4th order mesenteric arteries were mounted in a pressure myograph and the functional role of $\mathrm{K}_{\mathrm{IR}}, \mathrm{SK}_{\mathrm{Ca}}$ and $\mathrm{IK}_{\mathrm{Ca}}$ channels in modulating the myogenic response was explored using selective pharmacological tools.

Results: In normal buffer, arteries maintained their diameter at pressures above 40 $\mathrm{mmHg}$ whereas in $\mathrm{Ca}^{2+}$-free buffer, passive dilation was observed at each step demonstrating active myogenic tone development. Inhibition of $\mathrm{SK}_{\mathrm{Ca}}$ and $\mathrm{IK}_{\mathrm{Ca}}$ channels with either apamin (250 nM) or TRAM-34 (5 $\mu \mathrm{M})$, respectively, did not alter myogenic reactivity. In the presence of $50 \mu \mathrm{M} \mathrm{Ba}^{2+}$, myogenic reactivity was unaltered but with a higher concentration (100 $\mu \mathrm{M})$, myogenic reactivity was enhanced at lower pressures $(<60 \mathrm{mmHg})$. CyPPA $(5 \mu \mathrm{M})$, a positive modulator of $\mathrm{SK}_{\mathrm{Ca}}$ channels, and SKA$31(5 \mu \mathrm{M})$, an activator of $\mathrm{IK}_{\mathrm{Ca}}$ channels, each abolished the myogenic response. The effect of CyPPA was inhibited by $50 \mu \mathrm{M} \mathrm{Ba}^{2+}$ but a higher concentration of $\mathrm{Ba}^{2+}(100$ $\mu \mathrm{M} \mathrm{Ba}^{2+}$ ) was required to inhibit the effect of SKA-31.

Conclusions: Our findings indicate that the ability of pharmacological activators of $\mathrm{SK}_{\mathrm{Ca}}$ and $\mathrm{IK}_{\mathrm{C}_{\mathrm{a}}}$ channels to inhibit myogenic reactivity requires the engagement of KIR channels. 
Board: 3

Title: Distinct Regulation of Endothelial and Smooth Muscle KIR2.x Channel Pools by Membrane Lipids in Cerebral Arteries

Authors: Sancho M, Welsh DG

Address: Dept. of Physiology \& Pharmacology, Schulich School of Medicine, Western University, Canada

Abstract: In the cerebral circulation, inwardly rectifying potassium (KIR) channels are expressed in both endothelial and smooth muscle cells. This pattern of expression is unusual since vascular cells are electrically synchronized and it advocates that each pool could be differentially regulated, driving a distinct subset of physiological responses. This study defined the electrical and molecular properties of KIR2.x channels in cerebral arteries and determined the impact of phosphatidylinositol-bis-phosphate (PIP2) and cholesterol, in modulating each channel pool.

Endothelial and smooth muscle cells were freshly isolated from rat cerebral arteries; patch clamp electrophysiology and Q-PCR delineated KIR activity and expression, respectively. Our electrophysiological results highlight that a Ba2+-sensitive KIR current is present and stable in both cell types; Q-PCR noted that KIR2.x mRNA subunits were present in both endothelial and smooth muscle cells. Intriguingly, endothelial but not smooth muscle KIR channels were activated by PIP2 and inhibited by ATP depletion, neomycin, and PI kinase blockers. In striking contrast, smooth muscle but not endothelial KIR channel activity was augmented by cholesterol depletion ( $\beta$ methylcyclodextrin).

In summary, our observations indicate that while endothelial and smooth muscle both express KIR2.x channels, each pool is uniquely regulated by membrane lipids. A complete picture of the expression/regulation of KIR channels within the vascular wall will provide further advances in our understanding of their role in cerebral blood flow control in health and disease. 


\section{Board: 4}

Title: $\quad$ Establishing channelrhodopsin-2 for use in vascular optogenetics

Authors: Nils J. G. Rorsman ${ }^{1,2}$, Chau M. Ta ${ }^{1}$, Hannah Garnett ${ }^{1}$, Pawel Swietach ${ }^{3}$, Paolo Tammaro ${ }^{1,2}$

Address: $\quad{ }^{1}$ Department of Pharmacology, University of Oxford, Mansfield Rd, Oxford OX1 3QT, United Kingdom;

${ }^{2}$ OXION Initiative in Ion Channels and Disease, University of Oxford, Oxford, United Kingdom;

${ }^{3}$ Department of Physiology, Anatomy and Genetics, University of Oxford, Oxford OX1 3PT, United Kingdom

Abstract: Optogenetic control of electromechanical coupling of vascular smooth muscle is emerging as a powerful research tool with potential translation into new therapeutic strategies. The exact ionic mechanisms involved in this control, however, remain unexplained. Here we combine cell imaging, patch-clamp electrophysiology and arterial tension recordings to define these mechanisms in a wide range of light stimulations. Mice expressing a channelrhodopsin-2 variant (ChR2(H134R)) selectively in smooth muscle cells (SMCs) were generated. Isolated systemic and pulmonary SMCs obtained from these mice demonstrated depolarising, blue light induced whole-cell currents. The tone of isolated arteries could be finely controlled by varying the intensity of the light stimulus. This arterial response was sufficient to overcome the melanopsin-mediated light-depended arterial relaxation observed in the presence of contractile agonists. Ca2+ entry through voltage-gated $\mathrm{Ca} 2+$ channels and recruitment of plasmalemmal TMEM16A Cl- channels, but not activation of intracellular ryanodine receptors, were mandatory for ChR2(134R)-mediated arterial response to blue light at intensities lower than $\sim 0.1 \mathrm{~mW} / \mathrm{mm} 2$. Light stimuli of greater power caused a significant $\mathrm{Ca} 2+$ influx directly through $\mathrm{ChR2}$ (H134R) and provoked dramatic alkalinisation of intracellular $\mathrm{pH}$ of SMCs. This systematic analysis identified the range of light intensities that enabled optical control of arterial tone primarily through modulation of endogenous $\mathrm{CaV}$ channels while also limiting changes in $\mathrm{pHi}$. Within this identified range of light stimulations, the ChR2(H134R)-SM mice constitute a powerful experimental model to achieve accurate and tuneable optical voltage-clamp of SMCs and fine, graded control of arterial tone. 
Board: 5

Title: Different population of circulating T lymphocytes in obese patients undergoing bariatric surgery.

Authors: C. Agabiti Rosei ${ }^{1}$, C. Rossini ${ }^{1}$, F. Mittempergher ${ }^{2}$, A. Titi ${ }^{2}$, N. Portolani ${ }^{2}$, C. De Ciuceis ${ }^{1}$, S. Caletti ${ }^{1}$, MA Coschignano ${ }^{1}$, V. Trapletti ${ }^{1}$, E. Porteri ${ }^{1}$, P.Pileri ${ }^{1}$, E. Agabiti Rosei ${ }^{1}$, D. Rizzoni ${ }^{1,3}$.

Address: $\quad{ }^{1}$ Clinica Medica and ${ }^{2}$ Clinica Chirurgica, Department of Clinical and Experimental Sciences, University of Brescia, Italy; ${ }^{3}$ Istituto Clinico Città di Brescia, Division of Medicine, Brescia, Italy.

Abstract: Objective It has been previously demonstrated that $\mathrm{T}$ lymphocytes may be involved in the development of hypertension and microvascular remodeling, and that circulating $T$ effector lymphocytes may be increased in hypertension. In particular, Th1 and Th 17 lymphocytes may contribute to the progression of hypertension and microvascular damage while TREG lymphocytes seem to be protective. However, no data is avaliable about patients with severe obesity, in which pronounced microvascular alterations were observed. Design and Methods We have investigated 32 severely obese patients undergoing bariatric surgery, as well as 24 normotensive lean subjects and 11 hypertensive lean subjects undergoing an election surgical intervention. No sign of local or systemic inflammation was present in any subject or patient. A peripheral blood sample was obtained before surgery for assessment of CD4+ T lymphocyte subpopulations. Lymphocyte phenotype was evaluated by flow cytometry after 5 hour in vitro activation in order to assess T-effector and T-regulatory (TREG) lymphocytes. Subsets of TREGS were defined as follows: -TREGS recent thymic emigrants (RTE), directly derived from thymus: CD31+; -TREGS naïve: CCR7+CD45RA+; -TREGS central memory (CM): CCR7+CD45RA-; -TREGS effector memory (EM): CCR7-CD45RA-; -TREGS terminal differentiated effector memory (TDEM): CCR7-CD45RA+.

Results are summarized in the Table $\left({ }^{*} p<0.05, * * p<0.01, * * * p<0.001\right.$ vs. lean normotensives; $\# p<0.05, \# \# p<0.01, \# \# \# p<0.001$ vs. lean hypertensives). A marked reduction of several TREG subpopolations was observed in obese patients compared with controls, together with an increased in some T-effector cells.

Conclusion TREG lymphocytes are clearly reduced in severely obese patients, possibly contributing to the development of marked microvascular alterations previously observed in such a population.

\begin{tabular}{|l|l|l|l|}
\hline & Lean normotensives & \multicolumn{1}{|c|}{ Lean hypertensives } & \multicolumn{1}{c|}{ Obese patients } \\
\hline TREGs (\%) & $4.11 \pm 1.60$ & $4.64 \pm 1.66$ & $2.69 \pm 1.81^{* * \# \#}$ \\
\hline TREGs (abs number) & $45.4 \pm 24.3$ & $45.4 \pm 23.8$ & $27.3 \pm 21.1^{* * * \#}$ \\
\hline TREGs naive (\%) & $22.1 \pm 10.1$ & $18.1 \pm 13.1$ & $13.34 \pm 12.9^{* * *}$ \\
\hline TREGs naive (abs number) & $10.6 \pm 7.75$ & $9.71 \pm 8.87$ & $3.87 \pm 5.28^{*} * \# \#$ \\
\hline TREG CM (\%) & $32.3 \pm 13.8$ & $32.8 \pm 17.8$ & $22.7 \pm 15.2^{*} \#$ \\
\hline TREGs CM (abs number) & $14.7 \pm 10.2$ & $14.2 \pm 9.08$ & $6.10 \pm 8.08^{* * * \# \#}$ \\
\hline CD4+ EM (\%) & $24.4 \pm 9.96$ & $26.8 \pm 12.5$ & $34.1 \pm 13.3^{* * *}$ \\
\hline CD161+CD28+ (\%) & $86.2 \pm 28.5$ & $94.9 \pm 5.63$ & $97.2 \pm 5.39^{*}$ \\
\hline
\end{tabular}


Board: 6

Title: Shrinkage significantly influences morphometric evaluation of the wall to lumen ratio

Authors: Maria Bloksgaard ${ }^{1}$, Kristoffer Rosenstand ${ }^{1}$, Inger Nissen ${ }^{1}$, Niels Marcussen ${ }^{2}$, Jo G.R. De Mey $^{1,3}$

Address: University of Southern Denmark; ${ }^{1}$ Dept. Cardiovascular and Renal Research, Institute of Molecular Medicine, Odense, Denmark

Odense University Hospital; ${ }^{2}$ Dept. Clinical Pathology and ${ }^{3}$ Dept. Cardiac, Thoracic and Vascular Surgery, Odense, Denmark

Abstract: Structural remodeling of resistance arteries is predictive of future adverse cardiovascular events in hypertensive patients. Assessment of the classical remodeling indices, the media- and wall- to-lumen ratios ( $M: L$ and $W: L$, respectively), requires measurement of the dimensions of the arterial wall in isolated resistance arteries, studied ex vivo under standardized conditions. Traditionally, assessment of lumen perimeter, wall- and tunica media thickness has been performed in resistance arteries mounted in myographs. However, this is complicated in e.g. human pericardial resistance arteries not displaying a prominent external elastic lamina. In this work, we determined the impact of formalin fixation and paraffin embedding on resistance artery structure. We show that the resistance arterial wall shrinks significantly $(60 \%)$ during histological processing, with great impact on the morphometric determination of arterial wall thickness and wall to lumen ratio. A correction factor is proposed for retrieving the dimensions of the vital artery. 
Board: 7

Title: $\quad$ Overexpression of the CDK5 regulatory subunit p25 in endothelial cells promotes endothelial senescence and adverse arterial remodeling in arteries

Authors: Yumeng Guo, Andy WC Man, Bo Bai, Cheng Xu, Cuiting Luo, Aimin Xu, Paul M Vanhoutte, Yu Wang*

Address: State Key Laboratory of Pharmaceutical Biotechnology and Department of Pharmacology and Pharmacy, The University of Hong Kong, Hong Kong, China. *Email: yuwanghk@hku.hk

Abstract: Background- $\mathrm{P} 25$ is a 209-amino acid $\mathrm{COOH}$-terminal fragment of the regulatory subunit 1 (p35) for CDK5, a proline-directed serine/threonine kinase. Upregulation of p25 triggers a sustained activation of CDK5 and hyperphosphorylation of various substrates including SIRT1, the longevity regulator, in endothelial cells (1). The present study investigated the role of endothelial p25 in regulating endothelial senescence and adverse arterial remodeling.

Methods and Results- Overexpression of p25 in primary porcine aortic endothelial cells impaired cell proliferation and promoted senescence. Transgenic mice (EC-p25) with selective and controlled overexpression of p25 in vascular endothelial cells were generated. Compared to wild type littermates, arteries from 20-weeks old EC-p25 mice exhibited increased expression levels of various pro-inflammatory markers, such as $T N F \alpha, M C P 1, T G F-6, P$-selectin and MMP9. Under low-flow conditions, carotid arteries of EC-p25 mice showed significant neointima formation, as a result of hyperplasia and infiltration of vascular smooth muscle cells. The gene and protein expressions of soluble guanylyl cyclase (sGC) subunit $\beta 1$ were significantly downregulated in arteries of EC-p25, possible via the regulation of endothelial SIRT1.

Conclusion- The results implied a pathophysiological role of CDK5-p35/p25-SIRT1-sGC signaling axis in arterial remodeling and early vascular ageing will be discussed.

Acknowledgment: This work was financially supported by Hong Kong Health and Medical Research Fund 13142651.

\section{Reference:}

1. Bai B, Liang Y, Xu C, et al. CDK5-mediated hyperphosphorylation of SIRT1 contributes to the development of endothelial senescence and atherosclerosis[J].

Circulation, 2012: CIRCULATIONAHA. 112.118778. 


\section{Board: 8}

Title: $\quad$ Connexin40 mediates the conduction of afferent arteriolar vasoconstriction

Authors: Moller S, Holstein-Rathlou N-H and Sorensen CM

Address: University of Copenhagen, Dept. of Biomedical Sciences, Blegdamsvej 3C, DK-2200 Copenhagen, Denmark

Abstract: The renal vasculature expresses four isoforms of connexins (Cx), Cxs 37, 40, 43, and 45 . $\mathrm{Cx} 40$ is predominantly expressed in the juxtaglomerular apparatus which consists of the afferent and efferent arterioles, the glomerulus and the mesangial cells. Thus, Cx40 forms gap junctions, enabling intercellular communication in the juxtaglomerular apparatus. The tubuloglomerular feedback (TGF) mechanism regulates glomerular filtration by regulation of afferent arteriolar diameter. Activation of TGF is elicited in the juxtaglomerular apparatus and initiates a vasoconstriction of the afferent arteriole. Only the juxta-glomerular part of the afferent arteriole is in contact with the cells initiating the TGF-induced vasoconstriction but the constriction travels several hundred $\mu \mathrm{m}$ along the afferent arteriole. This conduction is presumably mediated by gap junctions.

We investigated the role of $\mathrm{Cx} 40$ in the TGF-induced vasoconstriction and the conduced vascular response. We show in isolated kidneys that $\mathrm{Cx} 40$ knockout (KO) mice have have no functional TGF. TGF was measured as decreases in afferent diameter during increases in perfusion pressure. Measurements were made both at a juxta-glomerular site where TGF is initiated and $100 \mu \mathrm{m}$ upstream. Furthermore, afferent arterioles lacking $\mathrm{Cx} 40$ have a significantly reduced conduction of vasoconstriction elicited by electrical stimulation. This stimulation mimics the vascular activation elicited by TGF. Also, Cx40 KO mice have no conduction of intercellular Ca2+increases in response to local electrical stimulation of interlobar arteries suggesting that reduced intercellular coupling significantly reduces conduction of vasoconstriction.

Our results show that $\mathrm{Cx} 40$ is involved in the signalling initiating TGF and in the conduction of the afferent vasoconstriction elicited by TGF. 
Board: 9

Title: $\quad$ Vitamin C deficiency impairs coronary artery muscarinic receptor-mediated vasoconstriction and smooth muscle phenotypic modulation

Authors: Gry Freja Skovsted ${ }^{1 *}$, Pernille Tveden-Nyborg ${ }^{1}$, Frederik Grell ${ }^{1}$, Karl Swärd ${ }^{2}$, Azra Alajbegovic $^{2}$, Sebastian Albinsson ${ }^{2}$ and Jens Lykkesfeldt ${ }^{1}$

Address: ${ }^{1}$ Department of Veterinary and Animal Sciences, Faculty of Health and Medical Sciences, University of Copenhagen, Ridebanevej 9, 1870 Frederiksberg C, Denmark. *Correspondence: gryfreja@sund.ku.dk; Tel.: +45-3533-7705

${ }^{2}$ Department of Experimental Medical Science, Lund University, Lund, Sweden

Abstract: Epidemiological studies have consistently found an association between vitamin C (vitC) deficiency and increased risk of cardiovascular disease, but the possible causal etiology has not been elucidated. In this study, we investigated the effect of vitC deficiency in guinea pigs on plasma biopterin status and vasomotor responses in coronary arteries; furthermore phenotypic modulation by vitC was studied in human coronary artery smooth muscle cells (hCASMCs). Dunkin Hartley female guinea pigs $(\mathrm{N}=32)$ were randomized to high $(1500 \mathrm{mg} / \mathrm{kg}$ diet) or low (0 to $50 \mathrm{mg} / \mathrm{kg}$ diet) vitC for 12 weeks. Coronary artery vasomotor responses to potassium, carbachol, sodiumnitroprusside (SNP), U46619, sarafotoxin6c (S6c) and endothelin-1 (ET-1) were recorded in a wire myograph. Plasma vitC and tetrahydrobiopterin were measured by HPLC. hCASMCs were cultured in vitC deficient medium and under physiological vitC concentrations. Expression of SMC differentiation/dedifferentiation markers were investigated by qPCR.

In vivo, vitC deficient animals displayed reduced tetrahydrobiopterin $(\mathrm{p}<0.001)$. Vasoconstrictor responses to carbachol were significantly decreased in vitC deficient coronary arteries independent of their general vasoconstrictor/vasodilator capacity $(p<0.001)$. Moreover, their carbachol-induced vasodilator responses correlated with coronary artery diameter $(p<0.001)$. Responses to SNP, potassium, S6c, U46619 and ET-1 were unaffected by vitC status. In vitro, vitC deficiency delayed proliferation and significantly modulated expression of differentiation/dedifferentiation markers in hCASMCs.

Our study shows that vitC deficiency induces functional down-regulation of vasoconstrictor muscarinic receptors in guinea pig coronary arteries. Furthermore, vitC deficiency impairs hCASMC proliferation and alters expression markers of SMC phenotypic modulation. Collectively, our data support that inadequate vitC status may play a role in impaired coronary artery function and during early coronary atherosclerosis. 
Board: $\quad 10$

Title: $\quad$ Regulation of vascular tone by Src kinase and its association with the $\alpha 2$ isoform $\mathrm{Na}+$, K+-ATPase

Authors: Christian Staehr, Elena Bouzinova, Christian Aalkjaer, Vladimir Matchkov

Address: Department of Biomedicine, Aarhus University, Denmark

Abstract: Background We have recently shown that mice with heterozygote G301R mutation of the $\alpha 2$ isoform $\mathrm{Na+}, \mathrm{K}+-\mathrm{ATPase}$ had reduced expression of this isoform in cerebral arteries. Simultaneous measurements of $[\mathrm{Ca} 2+] i$ and vasoconstriction in cerebral arteries in vitro suggested an increased $\mathrm{Ca} 2+$-sensitization of smooth muscles in $\alpha 2 \mathrm{G} 301+/-$ mice. Inhibition of tyrosine kinases (1 $\mu \mathrm{M}$ PP2) and Src kinase ( $2 \mu \mathrm{M}$ pNaKtide) abolished differences in Ca2+-sensitization. Western blotting suggested an increased Src activity at rest and after stimulation with U46619 (10 $\mu \mathrm{M})$ in $\alpha 2 \mathrm{G} 301+$ +cerebral arteries compared with wild type (WT). We here aimed to study neurovascular coupling (NVC) in $\alpha 2 \mathrm{G} 301+/-$ mice.

Method NVC in WT and $\alpha 2 \mathrm{G} 301+/$ - mice was accessed using confocal microscopy in brain slices loaded with CalciumGreen/AM. Changes in smooth muscle [Ca2+]i, and parenchymal arteriole diameter were measured in response to neuronal stimulation with electric field stimulation (EFS).

Results EFS induced Ca2+ waves in astrocytic endfeet, which were followed by relaxation of adjacent arterioles. The $\alpha 2 \mathrm{G} 301+/$ - mice had a larger maximal increase in diameter compared to WT mice $(7.83 \pm 0.87 \%, \mathrm{n}=8$ vs. $4.03 \pm 0.39 \%, \mathrm{n}=7 ; P=0.0012)$.

The decrease in smooth muscle [Ca2+]i associated with this relaxation was the same in both groups.

Conclusion Cerebral arterioles from the $\alpha 2 \mathrm{G} 301+/$ - mice showed increased dilations to neuronal activation despite similar changes in smooth muscle cell [Ca2+]i. We suggest that increased Src activity is responsible for $\mathrm{Ca2+-sensitization} \mathrm{of} \mathrm{smooth} \mathrm{muscles} \mathrm{and}$ the associated change in cerebrovascular functions of the $\alpha 2 \mathrm{G} 301+/$ - mice. 
Board: 11

Title: $\quad$ Nerve mediated vasorelaxation in rat mesenteric small arteries in vivo is $\beta$ adrenoceptor mediated.

Authors: Asger Maare Sondergaard, Aleksandra Mazur, Vladimir V. Matchkov and Christian Aalkjaer

Address: Department of Biomedicine, Aarhus University, Denmark

Abstract: We aimed to characterize nerve-mediated vascular relaxation in rat mesenteric arteries in vivo.

In intravital studies mesenteric small artery segments were imaged in a tissue chamber, where drugs could be applied. Perivascular adipose tissue (PVAT) was removed to provide a visual window. Rats were anesthetized with pentobarbital. The nerves were activated with electrical field stimulation (EFS) using increasing frequencies. The responses to EFS were TTX sensitive. Endothelium-dependent relaxation inhibitors ( $1 \mu \mathrm{M}$ TRAM-34, $3 \mu \mathrm{M}$ indomethacin, $50 \mathrm{nM}$ apamin and $100 \mu \mathrm{M}$ L-NAME) were present in the chamber through EFS relaxation experiment. Arteries incubated with $5 \mu \mathrm{M}$ guanethedine and preconstricted with $7 \mu \mathrm{M}$ methoxamine did not relax to EFS. In the next set of experiments the arteries were incubated with $140 \mu \mathrm{M}$ suramin and $1 \mu \mathrm{M}$ prazosin to block purinergic and $\alpha$ adrenergic receptors. In arteries preconstricted with $2 \mu \mathrm{M}$ U44619 EFS induced frequency-dependent relaxations, which were fully inhibited by $1 \mu \mathrm{M}$ propranolol $(26.8 \pm 10.5 \%$ of control relaxation, $n=5)$ while $1 \mu \mathrm{M}$ of the CGRP receptor blocker BIBN4096bs had no effect. EFS induced contractions in control and in the presence of 1 $\mu \mathrm{M}$ BIBN4096bs were similar ( $\Delta$ diameter at $16 \mathrm{~Hz}$ was $-95.0 \pm 12.5 \mu \mathrm{m}$ and $-98.22 \pm 9.2$ $\mu \mathrm{m}, \mathrm{n}=5$ under control conditions and in the presence of BIBN4096bs). In rat mesenteric small arteries without PVAT, EFS-induced relaxations are $\beta$ adrenoceptor but not CGRP-receptor mediated. 
Board: 12

Title: Identifying PTPN14-dependent mechanisms that influence clinical manifestations of Hereditary Hemorrhagic Telangiectasia

Authors: Ons Mamai, Kahlil Vaughan, Suprita Trilok, and Rosemary J. Akhurst.

Address: UCSF Helen Diller Family Comprehensive Cancer Center, 1450 3rd Street PO Box 589001, San Francisco, CA 94158-9001, USA

Abstract: Hereditary Hemorrhagic Telangiectasia (HHT) is a genetic disorder caused predominantly by loss of a single allele of ENG (HHT1) or ACVRL1 (HHT2). Global incidence is about 1 in 5,000. Clinical manifestations include cutaneous, mucosal and/or gastrointestinal ( $\mathrm{Gl}$ ) tract telangiectases that can cause severe epistaxis or $\mathrm{Gl}$ bleeding. Some patients (10-50\%) develop arteriovenous malformations (AVMs) in the lung, brain or liver. We previously showed that genetic variants of PTPN14 (PhosphoTyrosine Phosphatase Non-Receptor Type 14) genetically associate with the presence of lung AVMs. Homozygous loss of PTPN14 has also been reported to cause lymphedema due to lymph EC hyperplasia. Other studies, in tumor epithelial cells, show that PTPN14 can dephosphorylate ?-catenin, modulate HIPPO signaling and regulate tyrosine kinase receptor turnover through endosomal pathways. To investigate its role in ECs and its interactions with the endoglin/ACVRL1 axis, we studied the effect of PTPN14 knock down on differential expression of components of BMP9 and TGF-? signaling pathways in primary human umbilical artery ECs (HUAEC). PTPN14 expression had no effect on $\mathrm{pSmad} 2 / 3$ or $\mathrm{pSmad1} / 5 / 8$ activation but affected protein levels of VEGFR2 and EphrinB2. PTPN14 may thus act on a network of interacting signaling pathways, including endoglin and ACVRL1, by regulating cell surface receptor presentation and endocytic turnover. Studies are ongoing to address this issue in greater molecular detail. Elucidating the molecular mechanisms involved should contribute to a better understanding of the molecular pathology of HHT, and the regulation of angiogenesis versus stabilization of the vascular bed.

Keys words: PTPN14, BMP9, endothelial cell 
Board: 13

Title: $\quad$ Role of endothelial ENaC in LPS-induced permeability changes

Authors: M.Sternak ${ }^{1}$, Anna Bar $^{1,2}$, M.Adamski $^{1}$, Tasnim Mohaissen ${ }^{1}$, Brygida Marczyk $^{1}$, M.Stojak ${ }^{1}$, E. Martinez-Martinez ${ }^{3}$, F. Jaisser ${ }^{3}$, S.Chlopicki ${ }^{1,2}$

Address: ${ }^{1}$ Jagiellonian Centre for Experimental Therapeutics (JCET), Jagiellonian University, Krakow, Poland;

${ }^{2}$ Department of Experimental Pharmacology, Chair of Pharmacology, Medical College of the Jagiellonian University Medical College, Krakow, Poland

${ }^{3}$ INSERM U1138 Team 1, Integrative Physiology and Physiopathology Department, Centre de Recherche des Cordeliers INSERM, Paris, France

Abstract: The degree of $\mathrm{ENaC}$ activity seems to represent an important regulatory factor of endothelial phenotype.

The aim of this study was to analyze the role of $\mathrm{ENaC}$ in the regulation of endothelial permeability. $12 \mathrm{~h}$ after LPS, vascular permeability changes as the marker of endothelial dysfunction in vivo and ex vivo were measured in ENaCKO mice as compared to WT mice.

In vivo, endothelial permeability in BCA and LCA were assessed by MRI as the detection of $T$ relaxation time changes around vessel lumen in response to contrast agent administration. In vivo BBB permeability was also assessed by fluorescence changes in homogenates of perfused organs. For ex vivo studies, the permeability of FITC-dextran was evaluated in isolated mesenteric artery using pressure myograph system.

In vivo, after LPS administration, in ENaCKO mice shortening of T1 around arteries as well as the permeability increase in perfused organs were evident as opposed to WT mice. Ex vivo, FITC-dextran significantly penetrated through the vascular wall in isolated artery in ENaCKO mice in contrast to WT mice after LPS. Permeability changes were correlated with impaired endothelial response assessed in vivo by flow mediated dilatation measurements and the response to acetylcholine as well as lower intense of immunofluorescent staining for lectin. In ENaCKO mice LPS-induced permeability changes are potentiated in lung, brain and peripheral circulation and maybe linked to glycocalyx injury. Moreover, our data suggest that endothelial $\mathrm{ENaC}$ is mandatory for proper vascular endothelial function. Taken together endothelial permeability in endotoxemia is negatively regulated by endothelial $\mathrm{ENaC}$. 
Board: 14

Title: Impaired BKCa channel function in native vascular smooth muscle from humans with type 2 diabetes

Authors: Madeline Nieves-Cintrón ${ }^{1 *}$, Arsalan U. Syed ${ }^{1}$, Olivia R. Buonarati ${ }^{1}$, Robert R. Rigor $^{1}$, Matthew A. Nystoriak ${ }^{2}$, Debapriya Ghosh ${ }^{1}$, Kent C. Sasse ${ }^{3}$, Sean M. Ward $^{4}$, Johannes W. Hell ${ }^{1}$ and Manuel F. Navedo ${ }^{1 *}$

Address: ${ }^{1}$ Department of Pharmacology, University of California, Davis, CA 95616.

${ }^{2}$ Diabetes and Obesity Center, Department of Medicine, University of Louisville, Louisville, KY 40202.

${ }^{3}$ Sasse Surgical Associates, Reno, NV 89502.

${ }^{4}$ Department of Physiology and Cell Biology, University of Nevada, Reno, NV 89557.

Abstract: Large-conductance Ca2+-activated potassium (BKCa) channels are key determinants of vascular smooth muscle excitability. Functional coupling between localized intracellular $\mathrm{Ca} 2+$ release (i.e. Ca2+ sparks) and BKCa channels promotes vascular smooth muscle hyperpolarization and thereby opposes vasoconstriction. Suppressed BKCa channel function contributes to vascular complications in animal models of diabetes, but whether similar alterations occur in native vascular smooth muscle from humans with type 2 diabetes is unclear.

Here, we examined BKCa channel function in isolated arteries and freshly dissociated vascular smooth muscle cells from non-diabetic and type 2 diabetic patients. Arteries from diabetic patients exhibited reduced sensitivity to the selective BKCa inhibitor iberiotoxin. Consistent with altered BKCa channel function during diabetes, the amplitude and frequency of spontaneous BKCa currents, but not Ca2+ sparks, were lower in cells from diabetic patients. BKCa channels in cells from diabetic patients exhibited an apparent reduction in Ca2+ sensitivity and single-channel open probability. We also observed a decrease in tamoxifen sensitivity and in the coupling between the BKCa $\alpha$ and $\beta 1$ subunits, suggesting impaired BKCa $\beta 1$ function. These results indicate a reduced impact of BKCa channel function in vascular smooth muscle, which may contribute to the development of vascular complications in humans with type 2 diabetes. 
Board: 15

Title: Olive oil phenols dilate mesenteric resistance arteries in an endothelium independent manner.

Authors: Barberio $L^{1}$., Tropea $\mathrm{T}^{1}$., Muzzalupo $\mathrm{I}^{2}$., Mandalà $\mathrm{M}^{1}$.

Address: $\quad{ }^{1}$ Depart. of Biology, Ecology \& Earth Science, University of Calabria, Arcavacata di Rende, IT.

${ }^{2}$ Olivicoltura Research Center and Oil Industry, Arcavacata di Rende, IT.

Abstract: Several epidemiological studies have shown a role for Virgin Olive oil (VOO) consumption in the prevention of cardiovascular disease. Particularly, long-term intake of high doses of $\mathrm{VOO}$ reduces blood pressure and lowers the risk of developing hypertension. Some studies ascribe the hypotensive activity to phenols, which are minor components of VOO. The present study was designed to investigate the effect of VOO phenols on resistance arteries, and to identify the underlying molecular signals. Experiments were carried out on isolated pressurized rat mesenteric arteries by using a pressure myograph. Phenols induced concentration-dependent vasodilation, with a maximum effect of $83 \pm 7 \%$. A similar effect was observed in mesenteric arteries in which the endothelium was mechanically removed. Also, no significant differences were measured when the phenols were tested in presence of inhibitors for the following enzymes: 1) nitric oxide synthases, 2) cyclooxygenase, 3) guanylate cyclase and 4) adenylate cyclase. Interestingly, the phenol-induced vasodilation was abolished by depolarization and also was inhibited significantly in presence of the inhibitor of the big potassium channels (BK). In conclusion, VOO phenols are potent vasodilators of mesenteric resistance arteries. This effect is not mediated by the endothelium, but by a direct effect on smooth muscle cell via activation of BK potassium channels. 
Board: 16

Title: $\quad$ Exercise-induced interleukin 15 regulates insulin-dependent muscle blood flow

Authors: Beatriz CS Boa ${ }^{1}$, Femke PM Hoevenaars ${ }^{1}$, Zeineb Gam ${ }^{1}$, Erik van Poelgeest ${ }^{1}$, Fernanda M Ferrão ${ }^{2}$, Victor WM van Hinsbergh ${ }^{1}$, Eliete Bouskela ${ }^{2}$, Etto C Eringa ${ }^{1}$.

Address: ${ }^{1}$ Departments of Physiology, Institute for Cardiovascular Research, VU University Medical Center,

Amsterdam;

${ }^{2}$ Laboratory for Clinical and Experimental Research on Vascular Biology (BioVasc), Biomedical Center, State University of Rio de Janeiro, Brazil;

Abstract: Interleukin 15 (IL-15) is a muscle-derived hormone that regulates adipose tissue metabolism, mainly via actions of its receptor-alpha (IL-15R $\alpha$ ). Perivascular adipose tissue (PVAT) enhances insulin-induced vasodilatation in muscle arterioles, crucial to muscle insulin sensitivity. Here we investigated whether IL-15/IL-15R $\alpha$ alters PVAT increase of insulin-induced vasodilatation and muscle perfusion. Muscle perfusion was evaluated using contrast-enhanced ultrasonography (CEU) during hyperinsulinemic euglycaemic clamp and acute muscle contractions in control (C), exercised (EX) and IL$15 \mathrm{R} \alpha-/$ - male mice. Insulin-induced vasodilatation was studied in gracilis arterioles of EX or C mice in the pressure myograph with or without their own PVAT. Microvascular blood flow was substantially reduced in IL15R $\alpha$-/- mice compared to IL15R $\alpha+/+$ during hyperinsulinemia (Baseline:IL15R $\alpha+/+$ vs. IL15R $\alpha-/-$ $0.01 \pm 0.004$ vs. $0.01 \pm 0.001$ a.u.; hyperinsulinemia: ${ }^{*} 0.03 \pm 0.01$ vs. $+0.01 \pm 0.001$ a.u. $; P=0.0412$ vs. baseline and $+P=0.0002$ vs. IL15R $\alpha+/+)$. Exercise did not increase perfusion, but increased arteriolar responses to insulin $[(C) 0.4 \pm 3.6 \mathrm{vs}$. $(E X) * 29.5 \pm 13.3 \%$ of response; $p<0.01$ ]. Exercise also enhanced IL-15 and IL15R $\alpha$ mRNA in gastrocnemius and protein in gracilis muscle. Aortic PVAT expressed both IL-15 and IL15R $\alpha$. Acute pre-treatment of resistance arterioles with IL-15 (2ng/ml) blocks PVAT positive influence on insulin-induced vasodilatation. On the other, a higher dose $(200 \mathrm{ng} / \mathrm{ml})$ of this myokine did not impair this influence. Exercise decreased TNF$\alpha$, IL-1 $\beta$ and MCP-1 expression in gracilis PVAT. In conclusion, IL$15 R \alpha$ is crucial for normal muscle perfusion. Although exercise does not affect PVAT interaction with insulin-induced vasodilatation, it does increase arteriolar sensitivity to insulin and reduces inflammatory adipokines in PVAT. 
Board: 17

Title: $\quad$ The role of nitric oxide synthase in PVAT anti-contractility

Authors: Lauren Toms, Sophie N Saxton, Anthony M Heagerty.

Address: Institute of Cardiovascular sciences, University of Manchester, Manchester UK

Abstract: Perivascular adipose tissue (PVAT) surrounds the majority of blood vessels and there is an emerging body of evidence demonstrating a pivotal role of PVAT in vascular homeostasis. However, the mechanisms are unclear and loss of PVAT function in obesity may be contributing to the development of hypertension. Sympathetic innervation is likely to drive release of vasorelaxant factors by stimulating adipocyte $\beta 3$-adrenoceptors. This study aims to investigate the role of PVAT nitric oxide synthase (NOS) in the sympathetically-induced anti-contractile effect of PVAT. Electrical field stimulation (EFS) profiles $(0.1-30 \mathrm{~Hz}, 20 \mathrm{~V}, 0.2 \mathrm{~ms}$ pulse duration, $4 \mathrm{~s}$ train duration) of healthy and obese mouse mesenteric resistance arteries $(<250 \mu \mathrm{m},+$ /-PVAT) were characterised using wire myography. EFS induced a PVAT anti-contractile effect in healthy vessels, which was absent in obese mice. Inhibition of NOS in healthy PVAT using L-NMMA abolished the anti-contractile effect, whereas activation of NOS using histamine in obese PVAT restored the anti-contractile effect. Activation of $\beta 3$ adrenoceptors in healthy PVAT induced an enhancement of the anti-contractile effect, which could be blocked using NOS inhibition. Using immunohistochemistry, eNOS is the isoform present in healthy and obese PVAT, and expression appears unchanged. In conclusion, this study indicates that the healthy PVAT anti-contractile effect is dependent on NOS, and may mediate the effects of $\beta 3$-adrenoceptor activation in PVAT. In obesity, the anti-contractile effect is lost which may contribute to the development of hypertension; however function could be restored using NOS activation, indicating that NOS could be a useful pharmacological target in treating the vascular complications of obesity. 
Board: 18

Title: $\quad$ Exercise training improves anticontractile function of coronary arteries endothelium in rats with antenatal/early postnatal hypothyroidism

Authors: A.A. Shvetsova ${ }^{1,2,}$ D.K.Gaynullina ${ }^{1,2}$, S.I. Sofronova ${ }^{1}$, A.A. Borzykh ${ }^{1}$, E.K. Selivanova ${ }^{1,2,}$ I.V.Kuzmin ${ }^{1,2}$, A.A. Martyanov ${ }^{1,2}$, O.S. Tarasova ${ }^{1,2}$

Address: $\quad{ }^{1}$ SRC RF Institute for Biomedical Problems RAS, Moscow, Russia

${ }^{2}$ Faculty of Biology, Lomonosov Moscow State University, Moscow, Russia

Abstract: Objectives. We have recently shown that NO-mediated anticontractile effect of endothelium is eliminated in coronary arteries of adult rats suffered from antenatal/early postnatal hypothyroidism (Nitric Oxide 2017: 63:21-28). This study tested the hypothesis that exercise training would ameliorate such detrimental consequences of early thyroid deficiency.

Materials and Methods. Wistar dams received propylthiouracil (PTU, $7 \mathrm{ppm}$ ) in drinking water during gestation and two postpartum weeks; control dams received tap water. Six-week-old male offspring of PTU-dams was divided into sedentary and trained groups; the latter had 24-h access to running wheels. Eight weeks later coronary arteries were studied by wire myography. Anticontractile effect of NO was assessed by the effect of NOS inhibitor L-NNA on the contractile response to U46619. Blood T3/T4 and eNOS content were estimated by ELISA and Western blotting respectively.

Results. T3/T4 levels were depleted in the progeny of PTU-treated dams at $2 \mathrm{wk}$ age, but normalized at $4 w k$ age and thereafter. Coronary arteries of sedentary PTU offspring compared to control demonstrated higher basal tone and strongly reduced anticontractile effect of NO. In trained PTU offspring (run distance 8(5-22) km/week) coronary basal tone was reduced and NO-mediated anticontractile effect was stronger compared to the sedentary PTU group, despite unchanged NO-sensitivity (responses to $\mathrm{DEA} / \mathrm{NO}$ ). eNOS content in left ventricle was also increased in trained vs. sedentary PTU rats.

Conclusions. Transient thyroid deficiency during the antenatal/early postnatal period is followed by long-lasting coronary endothelial dysfunction, which can be corrected by voluntary exercise training. Supported by the Russian Science Foundation (grant N1415-00704). 
Board: $\quad 19$

Title: $\quad$ G Protein coupled receptor kinase 2 is essential for vasoconstrictor-stimulated arterial smooth muscle cell proliferation

Authors: Asma Alonazi ${ }^{1,2}$, Jonathon Willets ${ }^{1}$

Address: ${ }^{1}$ Department of Molecular and Cell Biology, University of Leicester, Henry Wellcome Building, Lancaster Road, Leicester LE1 9HN, UK.

${ }^{2}$ Department of Pharmacology and Toxicology, Pharmacy Collage, King Saud University, Riyadh, KSA.

Abstract: Vascular smooth muscle cell proliferation plays a key role in the development of hypertensive vascular remodelling, a process strongly associated with increased circulating vasoconstrictor concentrations, leading to continuous activation of their cognate Gaq-coupled/G protein-coupled receptors (GPCR). Furthermore, hypertension is associated with elevated $G$ protein coupled receptor kinase 2 (GRK2) expression in arterial smooth muscle cells (ASMC), which negatively regulates Gaq/GPCR signalling. As, GRK2 is implicated in cell proliferation, we utilised [3H]-thymidine incorporation assays and specific siRNA techniques to determine whether GRK2 played a role in vasoconstrictor-stimulated ASMC growth. SiRNA-mediated depletion of $>80 \%$ of GRK2 prevented angiotensinll or endothelin-1 (ET-1) stimulated ASMC proliferation over a 24 $\mathrm{h}$ period. Moreover, similar findings were observed following inclusion of the GRK2 inhibitor compound101. Since ERK signalling has previously been linked to vasoconstrictor GPCR-stimulated ASMC proliferation, we examined the effects of GRK2 depletion on ERK signalling. In negative-control (non-targeting) siRNA transfected cells angiotensinll or ET-1 induced a time-dependent increase in ERK phosphorylation, that peaked at $5 \mathrm{~min}$ and remained elevated for $>60 \mathrm{~min}$. Knockdown of GRK2 inhibited the peak, and virtually eliminated the prolonged phase of both angiotensinll and ET-1stimulated ERK phosphorylation. Inclusion of compound101 produced similar inhibition of agonist-stimulated ERK signals. These data suggest that the presence of GRK2 protein and its catalytic activity are required to mediate vasoconstrictor-stimulated ASMC proliferation. Moreover, since ERK signalling is known to mediate vasoconstrictor ASMC growth and as GRK2 is required for angiotensinll and ET-1mediated ERK signalling, this may explain why knockdown of GRK2 prevents vasoconstrictor-stimulated ASMC growth. 
Board: $\quad 20$

Title: $\quad$ Role of microsomal prostaglandin E synthase 1 in obesity-associated vascular damage

Authors: $\quad{ }^{1}$ Gonzalez-Amor $M,{ }^{1,2,3}$ García-Redondo AB, ${ }^{1}$ Rodrigues-Diez R, ${ }^{4}$ Beltrán LM, ${ }^{1}$ Ballesteros $C,{ }^{3,5}$ Martínez-Gonzalez J, ${ }^{1,2,3}$ Salaices $M,{ }^{1,2,3}$ Briones AM.

Address: $\quad{ }^{1}$ Departamento de Farmacología y Terapéutica, Facultad de Medicina, Universidad Autónoma de Madrid, Spain

${ }^{2}$ Instituto de Investigación Hospital Universitario La Paz (IdiPAZ), Madrid, Spain

${ }^{3} \mathrm{CIBER}$ de Enfermedades Cardiovasculares

${ }^{4}$ Servicio de Medicina Interna, Hospital Universitario La Paz, UAM, IdiPaz, Madrid, Spain.

${ }^{5}$ Instituto de Investigaciones Biomédicas de Barcelona (IIBB-CSIC), IIB-Sant Pau, Barcelona, Spain.

Abstract: Microsomal prostaglandin E synthase 1 (mPGES-1) is a cycloxygenase-2 downstream enzyme responsible for prostaglandin E2 (PGE2) production in inflammatory conditions. PGE2 is a powerful lipid mediator that influences the homeostasis of several organs and tissues including adipose tissue and vessels. The aim of the current study was to explore the role of mPGES-1 in obesity development and in obesityassociated vascular damage. We used mPGES-1+/+ and mPGES-1-/- mice exposed to normal or high fat diet (HFD, 21\% fat). HFD induced similar weight gain in mPGES-1+/+ and mPGES-1-/- with no differences in systolic blood pressure or heart weight within the four groups. HDF increased liver and epydidimal adipose tissue weight more in mPGES-1+/+ than in mPGES-1-/- mice. Moreover, HFD induced endothelial dysfunction in $\mathrm{mPGES}-1+/+$ but not in $\mathrm{mPGES}-1-/-$ mice. The mineralocorticoid receptor (MR) antagonist eplerenone, improved endothelium dependent relaxation in arteries from HFD-mPGES-1+/+ mice but not in mPGES-1-/- mice fed on HFD. Interestingly, HFD increased adipose tissue gene expression of aldosterone synthase (Cyp11b2) in mPGES-1+/+ but not in mPGES-1-/- mice. We tried to translate preclinical findings to human patients. Preliminary data showed positive correlations between mPGES-1, CYP11B2 and MR gene expression in visceral adipose tissue. Moreover, there is a positive correlation between pulse wave velocity (as a measure of vascular stiffness) and mPGES-1, CYP11B2 and MR gene expression. Conclusions: Our study suggests that mPGES-1-derived PGE2 is involved in obesity associated tissue damage and vascular endothelial dysfunction and stiffness probably through excessive aldosterone/MR pathway.

Key words: mPGES-1; aldosterone; obesity; vascular function.

Support: ISCIII-Fondo Europeo de Desarrollo Regional (FEDER): PI13/01488; MINECO:SAF 2016-80305-P; Roche-IDIPAZ; CIBERCV, COST-ADMIRE:BM1301. 
Board: 21

Title: Obligatory role for PKC $\delta$ in activation of store-operated TRPC1 channels in vascular smooth muscle

Authors: Miguel Martín-Aragón Baudel, Jian Shi \& Anthony Albert

Address: Molecular \& Clinical Sciences Research Institute, St. George's, University of London, UK.

Abstract: Stimulation of store-operated channels (SOCs) composed of TRPC1 in vascular smooth muscle cells (VSMCs) regulate cell contraction, proliferation and migration associated with vascular disease $(1,2)$. Therefore it is important to understand how TRPC1 SOCs are activated. Stimulation of TRPC1 SOCs and PKC phosphorylation by store depleting agents that do not increase intracellular $\mathrm{Ca}^{2+}$ concentration (e.g. BAPTA, TPEN) require PLC $\beta 1$ activity. This indicates that the PKC isoform involved requires diacylglycerol but is $\mathrm{Ca}^{2+-}$ sensitive - characteristics of the novel group of PKC isoforms (e.g. $\delta, \varepsilon, \eta, \theta$ ).

Since PKC $\delta$ has been linked to activation of SOCs in airway smooth muscle and is linked to regulating vascular tone (3), we focused on this isoform in activating TRPC1 SOCs.

The selective PKC $\delta$ inhibitor peptides, $\delta \mathrm{V} 1-1-\mathrm{TAT}$ and $\delta \mathrm{PKC} / 8-17$, inhibited whole-cell and single channel TRPC1 currents in freshly isolated mouse mesenteric artery VSMCs by over $80 \%$. Pre-treatment of mesenteric artery segments or single VSMCs with store depleting agents induced associations between TRPC1 and PKC $\delta$ at the plasma membrane using co-immunoprecipitation, immunocytochemical staining, and proximity ligation assay. Store depleting agents evoked relaxation of pre-contracted mesenteric arteries which were inhibited by $\delta \mathrm{V} 1-1-T a t$. Interestingly, in the presence of the large conductance $\mathrm{Ca}^{2+-}$ activated $\mathrm{K}^{+}$channel blocker iberatoxin, store depletion evoked vasoconstriction which was reduced by $\delta \mathrm{V} 1-1-T a t$.

These results indicate that PKC $\delta$ is likely to be the dominant PKC isoform involved in activating TRPC1 SOCs, and mediates store-operated changes in vascular reactivity.

1. Shi et al (2016) FASEB J 30, 702-715; 2. Shi et al (2017) J Physiol 595, 1039-1058

3. Garcia et al (2011) Channels 5, 210-214 
Board: 22

Title: Gender Differences in the Regulation of Renal Arteries from Normotensive and Hypertensive Rats

Authors: Hericka B. Figueiredo Galvao and lain Greenwood

Address: Vascular Biology Research Centre, Institute of Cardiovascular \& Cell Sciences, St. George's, London, United Kingdom.

Abstract: Introduction: Hypertension is a major risk factor for a number of cardiovascular diseases. Kv7 channels contribute to resting membrane potential in vascular smooth muscle cells and their role in vascular tone has been identified. However, their role in the female vasculature has not been investigated.

Objective: This study aims to compare the expression and function of Kv7 channels in the renal artery vasculature of female Wistar rats and SHRs.

Methods and Results: KCNQ1, KCNQ4, KCNE4 mRNA expression, as well as miRNA 153 and 133a in male and female renal arteries were determined by quantitative ReverseTranscription Polymerase Chain Reaction. Relative protein expression of Kv7.1, Kv7.4 and KCNE4 were determined using Western Blots normalised to $\beta$-actin. Isometric tension recordings were performed on renal arteries using Methoxamine, ML213, ML277, ANP and Isoprenaline. The contribution of Kv7 channels to Isoprenalinemediated vasorelaxation were investigated using the pan-Kv7 blocker Linopirdine. Male and female Wistar rats and SHRs expressed different levels of Kv7.1 and Kv7.4 which were discordant with mRNA transcript expression. Isometric tension recording report an increased sensitivity to ML213, ML277 and ANP in the female vasculature, and a decreased sensitivity to Methoxamine, compared to the male vasculature. Conclusions: This study provides evidence for the role of Kv7 channels in the male vasculature in addition to a differential expression and function of KV7 channels in the female vasculature. Moreover, the Isoprenaline-mediated vasorelaxations in the female renal artery suggests the involvement of additional Kv7 channels, not just Kv7.4, in $\beta$-adrenoceptor mediated vasorelaxations. 
Board: 23

Title: Angiotensin II promotes Kv7.4 channels degradation through reduced interaction with HSP90

Authors: Vincenzo Barrese, Jennifer B Stott, Hericka B Figueiredo, lain A Greenwood

Address: Vascular Research Centre, Molecular \& Clinical Sciences Research Institute, St George's University of London, Cranmer Terrace, SW17 ORE, London, United Kingdom

Abstract: Background. Voltage gated Kv7.4 potassium channels modulate arterial contractility, mediate responses to endogenous vasorelaxants, and are down-regulated in several arterial beds in different models of hypertension. Angiotensin II (Ang II), a key player in the pathogenesis of hypertension, affects the expression and trafficking of several classes of ion channels, including potassium channels. In this study we investigated whether Ang II altered the expression and function of vascular Kv7.4 channels.

Results. Western blot and quantitative PCR experiments revealed that in whole rat mesenteric artery (MA) Ang II incubation (1-7h) decreased Kv7.4 protein expression without reducing transcripts levels, and impaired the relaxation of MA induced by the Kv7.4 activator ML213 in wire-myography experiments. Immunofluorescence experiments showed that Ang II reduced Kv7.4 localisation at the plasma membrane in smooth muscle cells isolated from MA. Inhibition of proteasome with MG132 prevented Ang II-induced reduction of Kv7.4 levels measured by Western blot, and counteracted the impairment of ML213-induced relaxation caused by Ang II. Proximity Ligation Assays (PLA) showed that Ang II reduced the interaction of Kv7.4 with the molecular chaperone protein HSP90, and increased the interaction of KV7.4 with the E3 ubiquitin ligase CHIP. Inhibition of HSP90 with the geldanamycin analogue 17-AAG also decreased Kv7.4 protein levels and enhanced its interaction with CHIP. Conclusions. This study shows for the first time that Ang II downregulates Kv7.4 in a short time frame, by altering protein stability through a decrease of its interaction with HSP9O. This leads to an increased interaction with CHIP which drives to Kv7.4 degradation via the proteasome. 
Board: 24

Title: $\quad$ Silica nanoparticles induce ERK and Akt phosphorylation, resulting in impaired vascular function ex vivo.

Authors: Cai Astley ${ }^{1}$ Ali Shukur ${ }^{1}$, Fiona Wilkinson ${ }^{1}$, Yvonne Alexander $^{1}$, Debra Whitehead ${ }^{2}$, May Azzawi $^{1}$

Address: $\quad{ }^{1}$ Cardiovascular Research Group, School of Healthcare Science, Manchester Metropolitan University, M1 5GD

${ }^{2}$ School of Science and the Environment, Manchester Metropolitan University, Manchester, M1 5GD

\section{Abstract: Background:}

Silica nanoparticles (SiNPs) are widely reported to be biocompatible, however, their surface properties can affect their toxicity. We have previously demonstrated that SiNPs have no detrimental effect on conduit arterial function, but can influence small arterial function ex vivoz. The aim of the present study is to investigate the influence of SiNPs on vasodilation and examine signal transduction mechanisms underpinning impaired vascular function.

Methods: SiNPs were synthesised using a modified Stöber method and characterised using the Malvern Zetasizer and SEM. Pressure myography was used to assess the influence of SiNPs on endothelial-dependent vasodilator responses of small mesenteric arterial blood vessels from male Wistar rats ex-vivo following exposure to SiNPs at a concentration of $5.32 \times 10_{11} \mathrm{NPs} / \mathrm{mL}$. Western blotting was used to assess the expression of $\mathrm{p}$-ERK/p-Akt within mesenteric vessels after exposure to acetylcholine (ACh), SiNPs or ACh + SiNPs.

Results: SiNP size was confirmed as $85 \mathrm{~nm} \pm 13$. Zeta potential measurements confirmed SiNP stability in water and physiological salt solution. Exposure of mesenteric arteries to SiNPs ex-vivo resulted in a dosage-dependent attenuation of endothelial dependent dilator responses. We demonstrate that exposure to ACh leads to altered phosphorylation of ERK and Akt following SiNPs uptake.

Conclusions: SiNPs may attenuate ACh mediated dilation in small arteries by interfering with ERK/Akt signal transduction pathways. Our findings have important implications for the use of SiNPs for therapeutic intervention.

${ }_{1}$ N. Akbar, T. Mohamed, D. Whitehead, M. Azzawi (2011). Biocompatibility of amorphous silica nanoparticles: Size and charge effect on vascular function, in vitro. Biotechnology and Applied Biochemistry. 58(5), pp.353-362.

2 A. Shukur, D. Whitehead, A. Seifalian, M. Azzawi (2016). The influence of silica nanoparticles on small mesenteric arterial function. Nanomedicine (Lond). (16):213146. 
Board: 25

Title: Dietary Inorganic Nitrate Supplementation Improves Vascular Function in Pregnant eNOS-/- Mice

Authors: Teresa Tropea, Mark Wareing, Susan Greenwood, Colin Sibley, Elizabeth Cottrell

Address: St Mary's Hospital, University of Manchester, Manchester

Abstract: Introduction

The key pathology underlying fetal growth restriction (FGR) is uteroplacental insufficiency, involving vascular dysfunction and/or impaired nutrient transport. Vascular tone in the placenta is critically regulated by nitric oxide (NO), and aberrant NO production or action is associated with FGR in both humans and animal models. Mice deficient in endothelial nitric oxide synthase (eNOS-/- mice) exhibit reduced uteroplacental blood flow and FGR. Treatment with dietary nitrate, providing an exogenous source of NO, can improve blood flow and vascular function in nonpregnant individuals. We hypothesised that dietary nitrate supplementation may improve uteroplacental function in eNOS-/- mice.

Methods: Pregnant C57BI/6J (WT) and eNOS-/- mice were supplemented with nitratecontaining beetroot juice (BR+, $\sim 1 \mathrm{mmol} / \mathrm{kg} /$ day nitrate) in the drinking water from embryonic day (E)12.5. Control mice received either normal drinking water or an equivalent dose of nitrate-depleted 'placebo' juice, (BR-). At E18.5, maternal uterine and mesenteric arteries (UtA and MesA, respectively) were isolated. Contraction to the thromboxane mimetic U46619 and endothelium-dependent relaxation to acetylcholine (Ach) were assessed using wire myography.

Results:

Supplementation of pregnant eNOS-/- mice with BR+ improved maternal UtA vascular function. UtA of BR+ mice exhibited increased relaxation to Ach $(p<0.01)$ compared with water-treated controls. Vascular function was not altered by treatments in WT mice, nor in mesenteric arteries of eNOS-/- or WT mice.

Conclusions:

Supplementation with dietary nitrate improves vasodilation within the reproductive vascular system in the pregnant eNOS-/- mouse. 
Board: 26

Title: Enhanced interstitial fluid drainage in the hippocampus of spontaneously hypertensive rats

Authors: $\quad$ Erik N.T.P. Bakker ${ }^{1}$, Beatrice Bedussi ${ }^{1}$, Daphne M.P. Naessens ${ }^{1}$, Judith de Vos $^{1}$, Micha Wilhelmus ${ }^{2}$, Edo Richard ${ }^{3}$, and Ed vanBavel ${ }^{1}$

Address: ${ }^{1}$ Department of Biomedical Engineering and Physics, Academic Medical Center, Amsterdam, The Netherlands

${ }^{2}$ Department of Anatomy and Neurosciences, Neuroscience Campus Amsterdam, VU medical center, Amsterdam, The Netherlands

${ }^{3}$ Department of Neurology, Academic Medical Centre, Amsterdam, Netherlands;

Department of Neurology, Radboud University Medical Centre, Nijmegen, Netherlands.

Abstract: Hypertension is associated with cognitive decline and various forms of dementia, including Alzheimer's disease. Animal models of hypertension show increased accumulation of amyloid $\beta$ in the brain, a hallmark of Alzheimer's disease. Blood vessels may contribute to clearance of amyloid $\beta$, through transport across the bloodbrain barrier, as well as drainage along paravascular pathways. Here we studied the impact of hypertension on clearance of tracers from the brain, as a surrogate marker for amyloid $\beta$ removal. We used spontaneously hypertensive rats (SHR) and normotensive Wistar Kyoto rats (WKY). At 8 months of age, high (500 kD) and low (3 $\mathrm{kD}$ ) fluorescent molecular weight tracers released passively into the hippocampus showed a drastically enhanced spreading in SHR. Tracer spreading was inhomogeneous, with accumulation at border zones were interstitial fluid is separated from cerebrospinal fluid, around arteries, and towards a specific anatomical location, the stratum lacunosum moleculare. These locations stained positively for the astrocyte marker GFAP, and the water transporter aquaporin 4. Despite enhanced dispersion, clearance of tracers from the brain was not affected in SHR. In conclusion, these data indicate enhanced bulk flow of interstitial fluid in the hippocampus of hypertensive rats. Interstitial fluid drains along astrocytes towards the cerebrospinal fluid compartment, which extends along arteries as paravascular spaces into the brain. This bulk flow may lead to sieving of high molecular weight solutes, which could lead to a local increase in the concentration of waste products and potentially promotes the aggregation of amyloid $\beta$.

This project has received funding from the Internationale Stichting Alzheimer Onderzoek (ISAO) and from the European Union's Seventh Framework Programme for research technological development and demonstration under Grant agreement no. 606998. 


\section{Selected abstracts for oral presentation}

Title: $\quad$ TRPM3 dependent-vasodilation in resistance arteries

Authors: Lucía Alonso-Carbajo ${ }^{1,2}$ Yeranddy A. Alpizar ${ }^{1}$, Justyna Startek ${ }^{1}$, Jose Ramón LópezLópez $^{2}$, Maria Teresa Pérez-García ${ }^{2}$ and Karel Talavera ${ }^{1}$

Address: ${ }^{1}$ Laboratory of Ion Channel Research, Dept. Cellular and Molecular Medicine. KU Leuven, Leuven, Belgium

${ }^{2}$ Dpto de Bioquímica, Biología Molecular y Fisiología e Instituto de Biología y Genética Molecular (IBGM), Universidad de Valladolid y CSIC, Valladolid, Spain

Abstract: The vascular tone is determined by a complex interplay of vasodilator and vasoconstrictor stimuli that modulate the contractile state of vascular smooth muscle cells (VSMCs). Activation of the cation channel Transient Receptor Potential Melastatin 3 (TRPM3) has been shown to induce contraction of VSMCs in aorta. However, the contribution of this channel to the vascular tone in resistance arteries remains unknown. Real-time qPCR and immunochemistry experiments showed Trpm3 expression in mesenteric arteries isolated from wild type (WT) C57BL/6J mice. Myography experiments carried out in intact pressurized mesenteric arteries from WT mice showed that the TRPM3 agonist PS induces vasodilation at concentrations above $\sim 5 \mu \mathrm{M}$. However, PS only induced vasodilation above $10 \mu \mathrm{M}$ in preparations from Trpm3 knockout (KO) mice. The presence of the CGRP receptor antagonist BIBN 4096 indicated that the TRPM3-mediated effect of PS entails CGRP release from perivascular nerve endings. The effect of $10 \mu \mathrm{M}$ PS was inhibited to about $50 \%$ by the combination of potassium channel blockers ( $500 \mathrm{nM}$ paxilline, $10 \mu \mathrm{M}$ correolide and $50 \mathrm{nM}$ stromatoxin). Electrophysiological recordings and calcium imaging experiments in freshly isolated mesenteric VSMCs, revealed that basal currents and intracellular calcium concentration are not sensitive to PS $(10 \mu \mathrm{M}$ and $30 \mu \mathrm{M})$ and that PS has no effect on potassium currents. Our data indicates that activation of TRPM 3 channels in perivascular sensory nerves induces CGRP release, which leads to activation of potassium channels in smooth muscle cells, resulting in dilation of mesenteric arteries. These findings support a potential role of TRPM3 in response to endogenous agonists that could be produced in pathophysiological scenarios and the implication of this channel in neurogenic inflammation. 
Title: $\quad \mathrm{K}+$ transport abnormalities in cerebral arteries disturb neurovascular coupling in Familial Hemiplegic Migraine type 2 (FHM2)

Authors: C. Stæhr ${ }^{1}$, R. Rajanathan1, S. Sandow ${ }^{2}$, C. Aalkjaer1, V. Matchkov ${ }^{1}$

Address: $\quad{ }^{1}$ Department of Biomedicine, Aarhus University, 8000 Aarhus, Demark

${ }^{2}$ Faculty of Science, University of the Sunshine Coast, Qld 4558, Australia

Abstract: FHM2 is associated with point-mutations (G310R) in the $\alpha 2$ isoform Na, K-ATPase. Behavioural and neuronal abnormalities of heterozygous mice bearing the G301R mutation are recently characterized. The mutation leads to $50 \%$ reduction in the $\alpha 2-$ $\mathrm{Na}$, K-ATPase expression in cerebral arteries.

We have questioned whether FHM2 mice have abnormal neurovascular coupling in comparison with wild type mice (WT).

These abnormalities were assessed in vitro in an isometric myograph, in situ in brain slices with confocal microscopy and in vivo using Laser Speckle imaging.

Pre-constricted middle cerebral arteries from FHM2 mice had enhanced relaxation to increase in [K+]out ( 7-18 mM), which was associated with larger smooth muscle hyperpolarization in FHM2 than in WT. Inhibition of the $\alpha 2-\mathrm{Na}$,K-ATPase by $10 \mu \mathrm{M}$ ouabain was without any significant effect on the $\mathrm{K}+$-induced relaxation of arteries from FHM2 mice, and only slightly suppressed the relaxation in WT. The K+-induced relaxation was strongly inhibited by $30 \mu \mathrm{M} \mathrm{BaCl} 2$ suggesting a main role of the inwardrectifying $\mathrm{K}+$ channels (Kir). This was not associated with an increased expression of vascular Kir. In brain slices from FHM2 mice, neuronal electric field stimulation induced enhanced vasodilation of supplying parenchymal arteries. In vivo whisker stimulation induced increased elevation of blood flow in sensory cortex of FHM2 mice in comparison with WT.

Thus, the FHM2-associated mutation of the $\alpha 2-\mathrm{Na}, \mathrm{K}-\mathrm{ATP}$ ase leads to a Kir dependent, increased relaxation of parenchymal arterioles during repeated neuronal excitation. This dysfunction in neurovascular coupling might be involved in hyperperfusion associated with the headache stage of migraine attack. 
Title: $\quad[\mathrm{Ca} 2+]$ changes in axons in rat mesenteric arteries: relation to noradrenaline release and contraction

Authors: Makhala M. Khammy ${ }^{1,2}$, Avelino Ferreira ${ }^{2}$, Christian Aalkjær ${ }^{2}$

Address: ${ }^{1}$ Department of Biomedical Sciences, Faculty of Health and Medical Sciences, University of Copenhagen, Copenhagen, Denmark.

${ }^{2}$ Department of Biomedicine, Aarhus University, Aarhus, Denmark.

Abstract: Sympathetic vascular tone is tightly regulated at the level of the neurovascular junction. While it is known that presynaptic receptors modulate neurotransmitter release and that neurotransmitter release is coupled to varicose $\mathrm{Ca} 2+$ concentration $([\mathrm{Ca} 2+] \mathrm{v})$, there is no direct evidence as to whether presynaptic receptors modify transmitter release through modification of $[\mathrm{Ca} 2+] \mathrm{v}$.

This study investigated the relationship between [Ca2+]v, endogenous noradrenaline release and force development in response to electrical field stimulation (EFS) in rat mesenteric arteries using a combination of continuous noradrenaline-amperometry, wire myography and imaging of axons selectively loaded with the Ca2+ sensitive dye, Oregon GreenTM-dextran. EFS increased [Ca2+]v, stimulated noradrenaline release and elicited vasoconstriction.

The N-type voltage-gated calcium channel inhibitor, $\omega$-Conotoxin GVIA (100 nM), markedly diminished EFS-induced noradrenaline release and vasoconstriction despite only partially inhibiting the EFS-induced rise in [Ca2+]v. This suggests that only a fraction of the [Ca2+]v increase following nerve activation is associated with noradrenaline release. Inhibition of voltage-gated potassium channels with 4amminopyridine $(10 \mu \mathrm{M})$ potentiated [Ca2+]v, noradrenaline release and force development to EFS. Similar responses were observed with the $\alpha 2$-adrenoceptor antagonist yohimbine $(0.3 \mu \mathrm{M})$, although yohimbine failed to potentiate force development. Notably, the potentiating effect of 4-amminopyridine and yohimbine on noradrenaline release was not greater than either agent alone; suggesting that $\alpha 2$ adrenoceptor-mediated inhibition of neurotransmitter release functions via the inhibition of voltage-gated potassium channels.

In conclusion, we have demonstrated how pharmacological intervention modifies norepinephrine release through modification of $[\mathrm{Ca} 2+] \mathrm{v}$. The protocols developed will be useful for assessing neurovascular function in conditions with sympathetic dysfunction. 
Title: Media-to-lumen ratio and contractility of resistance arteries from cardiovascular disease patients.

Authors: Jo G. R. De Mey ${ }^{1,2}$, Thomas M. Leurgans ${ }^{1}$, Katrine Nissen ${ }^{1}$, Susanne Hansen ${ }^{1}$, Inger Nissen ${ }^{1}$, Kristoffer Rosenstand ${ }^{1}$, Akhmadjon Irmukhamedov ${ }^{2}$, Lars P. Riber ${ }^{2}$, Lars M. Rasmussen $^{3}$, Maria Bloksgaard ${ }^{1}$.

Address: ${ }^{1}$ Dept. Cardiovascular and Renal Research, Institute of Molecular Medicine, University of Southern Denmark, Odense, Denmark; ${ }^{2}$ Dept. Cardiac, Thoracic and Vascular Surgery and ${ }^{3}$ Dept. Clinical Biochemistry and Pharmacology, Odense University Hospital, Odense, Denmark.

Abstract: Background. The media-to-lumen ratio of resistance arteries is increased in hypertension and diabetes and an independent predictor of cardiovascular events. We tested the hypothesis that in cardiovascular disease patients, the contractile reactivity of resistance arteries increases with the size of the tunica media. Methods. Resistance artery segments $(n=503)$ were isolated from biopsies of the parietal pericardium obtained during cardiothoracic surgeries $(\mathrm{N}=128)$ and their maximal contractile response and structural properties were determined. Results. Lumen diameter, media cross-sectional area and media-to-lumen ratio averaged $188(155-218) \mu \mathrm{m}, 6175$ $(3746-8718) \mu \mathrm{m}^{2}$ and $5.32(4.43-6.17) \%$ (median (interquartile range)), respectively. Contractile responses to high $\mathrm{K}^{+}$, thromboxane $\mathrm{A}_{2}$ analogue plus endothelin-1 (1.48 $(0.91-2.27) \mathrm{N} / \mathrm{m}$ ) were positively related to arterial lumen diameter and media thickness (both $\mathrm{P}<0.0001)$ but not to media-to-lumen ratio $(\mathrm{P}=0.0854)$. In diabetics $(\mathrm{N}=24)$ compared to all non-diabetics, the media-to-lumen ratio was larger (6.03 \pm 1.47 versus $5.29 \pm 1.50 \%, \mathrm{P}=0.0371$ ) but this was not accompanied by a significantly larger contraction ( $1.89 \pm 1.15$ versus $1.62 \pm 0.95 \mathrm{~N} / \mathrm{m})$. Contraction normalized to media volume varied more than 7 -fold independently from classical patient characteristics except for small positive effects of systolic blood pressure and $\beta$-blocker treatment and a negative influence of hyperglycemia. Patients with a comparatively high normalized resistance artery contractility were younger, had a higher diastolic blood pressure and received more frequent $\beta$-blocker treatment. Conclusion. Contractile responses of resistance arteries from cardiovascular disease patients increase with the volume of the arterial smooth muscle but not with the media-tolumen ratio. The link between resistance artery media-to-lumen ratio and the risk for cardiovascular events may not involve resistance artery constriction. The structure and function of resistance artery smooth muscle are differentially influenced by risk factors and drug treatments. 
Title: Mechanisms of perivascular adipose tissue dysfunction in obesity and restoration using exercise

Authors: Sophie N Saxton ${ }^{1}$, Robert G Aldous1, Sarah B Withers ${ }^{1,2}$, Jacqueline Ohanian ${ }^{1}$, Anthony M Heagerty ${ }^{1}$.

Address: ${ }^{1}$ Institute of cardiovascular sciences, University of Manchester, Manchester ${ }^{2}$ BRC, Environment and Life Sciences, The University of Salford, United Kingdom

Abstract: Healthy perivascular adipose tissue (PVAT) exerts an anti-contractile effect on resistance arteries which is vital in regulating vascular tone. Activation of adipocyte $\beta_{3}$ adrenoceptors by the sympathetic nerve-derived noradrenaline may be implicated in this effect. Accordingly, we investigated sympathetic nerve stimulation within PVAT. Electrical field stimulation (EFS) profiles of murine mesenteric arteries $(<250 \mu \mathrm{m},+/$ PVAT) from healthy, obese, and exercised obese mice were characterised using wire myography $(0.1-30 \mathrm{~Hz}, 20 \mathrm{~V}, 0.2 \mathrm{~ms}$ pulse, $4 \mathrm{~s}$ train). During EFS PVAT elicited a reproducible anti-contractile effect, which was lost in obesity. Pharmacological sympathetic denervation using 6-hydroxydopamine abolished the anti-contractile effect. $\beta_{3}$-adrenoceptor function was investigated using the agonist $C L-316,243$ and antagonist SR59203A. SR59230A reduced the anti-contractile effect in health; however CL-316,243 could not restore function in obesity. Using immunohistochemistry, expression of $\beta_{3}$-adrenoceptors was reduced in obesity. Exogenous application of adiponectin induced vasodilation of healthy vessels, and a blocking peptide for adiponectin receptor-1 significantly reduced the anti-contractile effect of PVAT in health. However, in obese vessels exogenous adiponectin no longer exerted a vasodilator effect. In obese exercised mice, the PVAT anti-contractile was restored, and the development of hypertension and type-Il diabetes was reversed, although the mice remained obese. Exercise increased $\beta_{3}$-adrenoceptor expression, and restored the vasodilator effects of adiponectin. In conclusion, sympathetic nerve stimulation elicits an anti-contractile effect via activation $\beta_{3}$-adrenoreceptors and release of adiponectin. This effect was lost in obesity, which may contribute to development of hypertension and type-II diabetes. Exercise restored PVAT dysfunction in obesity, and normalised glucose and blood pressure independent of weight loss. 
Title: $\quad$ Caveolae Couple CaV3.2 to Ca2+ spark and STOC Production in Vascular Smooth Muscle

Authors: Ahmed M. Hashad ${ }^{1}$, Osama F. Harraz ${ }^{2}$, Suzanne E. Brett ${ }^{3}$, Mario Kassmann ${ }^{4}$, Maik Gollasch ${ }^{4}$, Donald G. Welsh ${ }^{1,3}$

Address: $\quad{ }^{1}$ Dept. of Physiology \& Pharmacology, Hotchkiss Brain and Libin Cardiovascular Institutes, University of Calgary, Alberta, Canada.

${ }^{2}$ Dept. Pharmacology, University of Vermont, Burlington, VT.

${ }^{3}$ Dept. Physiology \& Pharmacology, University of Western Ontario, London, Ontario, Canada.

${ }^{4}$ Charité University Medicine, Experimental and Clinical Research Centre, Berlin, Germany.

Abstract: CaV3.2 is integral to Ca2+ spark generation and the opening of large conductance $\mathrm{Ca} 2+$-activated $\mathrm{K}+(\mathrm{BKCa})$ channels, in the form of spontaneous transient outward current (STOC). This study examined whether the localization of CaV3.2 to lipid rafts "caveolae" is essential for successful spark initiation in mouse mesenteric smooth muscle cells. Proximity ligation assay (PLA) confirmed the close apposition of CaV3.2 with caveolin 1, a key constitutive protein within caveolae. Patch clamp electrophysiology subsequently revealed that caveolae disruption with methyl $\beta$ cyclodextrin (MBCD) specifically attenuated CaV3.2 current and BKCa channel activity in wild type and not CaV3.2-/- mice. Building on these observations, CaV3.2 blockade failed to modify Ca2+ sparks/STOC production in caveolin 1 knockout mice, despite continued expression of T-type Ca2+ channels. Further, pressure myography revealed that Ni2+ (CaV3.2 blocker)-induced constriction, a functional index of BKca-mediated feedback, was absent in caveolin 1-/- mice. Without caveolin 1 expression, CaV3.2 channels no longer co-localized with ryanodine receptors (PLA). In conclusion, the distinct localization of CaV3.2 channels within membrane caveolae provides effective coupling between the T-type channels, $\mathrm{Ca} 2+$ sparks production and arterial tone regulation. 
Title: Interaction between pressure-induced myogenic tone and TRPV4-mediated vasodilation in the rat cremaster muscle artery.

Authors: Timothy V. Murphy ${ }^{1}$, T. Hilton Grayson ${ }^{1}$, Shaun L. Sandow ${ }^{1,2}$

Address: $\quad{ }^{1}$ Physiology, School of Medical Sciences, University of NSW, Sydney, NSW Australia; ${ }^{2}$ Inflammation and Healing Cluster, Faculty of Science, Health, Education and Engineering, University of the Sunshine Coast, Sippy Downs, Qld, Australia.

Abstract: Transient receptor potential (TRP) channels are a broad class of ion channels with emerging roles in vascular physiology. The TRP vanilloid-4 (TRPV4) subtype induces vasodilation in many vessel types and is thought to be activated by 11,12-epoxyeicosatrienoic acid (11,12-EET). The present study investigated the interaction between TRPV4 and pressure-induced myogenic tone in the rat isolated cremaster muscle artery. Immunofluorescence studies showed TRPV4 to be predominantly expressed in the smooth muscle cells of the artery, with low expression in the endothelium. The TRPV4 activator GSK 1016790A (GSK) caused a concentration-dependent relaxation of arteries with decreasing potency over the pressure range $50-70-120 \mathrm{mmHg}$ ( $\mathrm{pEC} 50$ $9.03 \pm 0.18,8.06 \pm 0.07,7.31 \pm 0.15$ respectively, $n=5$ for each). Similar pressuredependent decreases in vasodilator potency were observed with 11,12-EET and adenosine. In contrast, the degree of pressure-induced myogenic tone did not alter relaxation of the arteries induced by acetylcholine, an endothelium-dependent vasodilator agent. The TRPV4 antagonist HC-067047 (HC; $0.3 \mu \mathrm{M})$ inhibited responses to GSK at each pressure, but $\mathrm{HC}$ inhibited responses to $11,12-\mathrm{EET}$ in vessels maintained at $50 \mathrm{mmHg}$ only, and adenosine-induced vasodilation at 50 and $70 \mathrm{mmHg}$ only. $\mathrm{HC}$ did not affect responses to acetylcholine at any pressure. The putative 11,12-EET antagonist 14,15-EE(Z)E $(10 \mu \mathrm{M})$ reduced GSK-induced vasodilation in vessels maintained at $50 \mathrm{mmHg}$ only. These findings suggest increasing pressure-induced myogenic tone inhibits both TRPV4-mediated vasodilation and the ability of 11,12-EET and adenosine to activate TRPV4 in vascular smooth muscle. 
